\title{
Macroscopic indicators of fault diagnosis and ageing in electrochemical double layer capacitors
}

\author{
Dimitri Torregrossa $^{\mathrm{a}, *}$, Kathryn E. Toghill ${ }^{\mathrm{b}, \mathrm{c}}$, Véronique Amstutz ${ }^{\mathrm{b}}$, Hubert H. Girault ${ }^{\mathrm{b}}$, \\ Mario Paolone ${ }^{a}$ \\ a Distributed Electrical System Laboratory at Swiss Federal Institute of Technology in Lausanne, Switzerland \\ ${ }^{\mathrm{b}}$ Laboratory of Physical and Analytical Electrochemistry, Ecole Polytechnique Fédéral de Lausanne, Switzerland \\ ${ }^{\mathrm{c}}$ Department of Chemistry, Lancaster University, Lancaster, UK
}

\section{A R T I C L E I N F O}

\section{Article history:}

Received 5 January 2015

Received in revised form 29 April 2015

Accepted 1 May 2015

\section{Keywords:}

Supercapacitors

Fault diagnosis

Recovering phase

Accelerated ageing phase

\begin{abstract}
A B S T R A C T
The ageing behaviour of electric double layer (EDLC) supercapacitors during over-temperature and overvoltage stresses is described. Specific experimental tests were carried out in order to reproduce, under laboratory controlled conditions, the above-mentioned stresses. The obtained results are discussed and interpreted to define indicators that distinguish between over-temperature and over-voltage faults occurring in these devices. For each stress type post-mortem examinations of the EDLCs were performed to observe any specific physical damage. These examinations have been used to define the fundamental assumptions of the proposed fault indicators. Three fault indicators were identified and related to the evolution of the following SC parameters: (i) the equivalent series resistance, (ii) capacitance and (iii) decay voltage during the relaxation phase. Additionally, new accelerated ageing and recovering phases were detected during combined high-pulsed power cycling and life endurance stresses.
\end{abstract}

() 2015 Elsevier Ltd. All rights reserved.

\section{Contents}

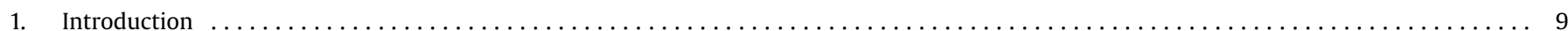

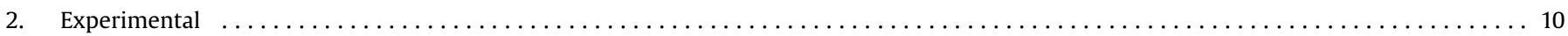

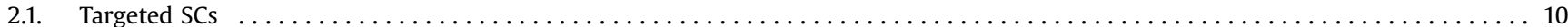

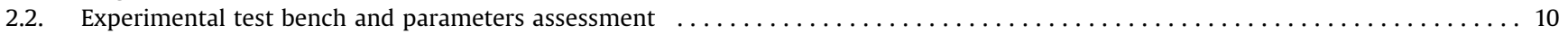

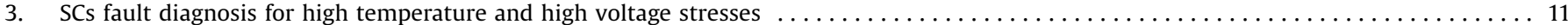

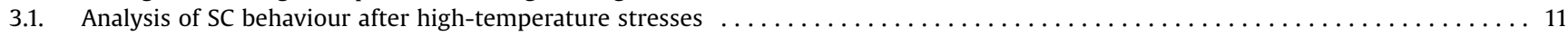

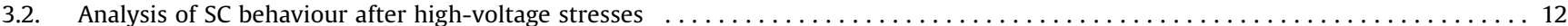

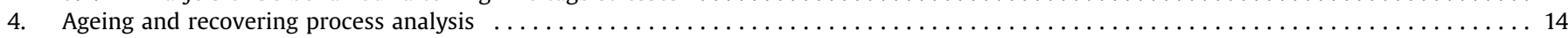

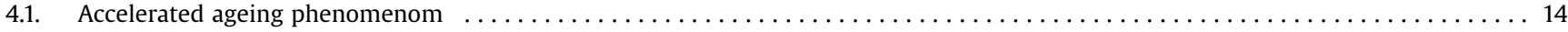

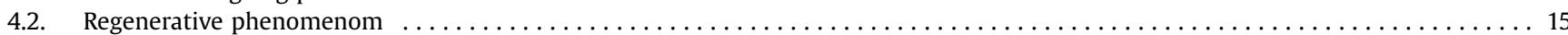

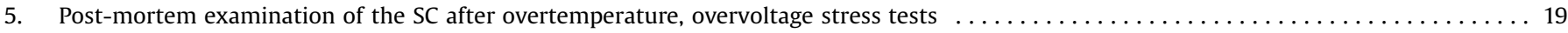

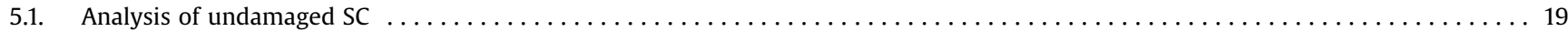

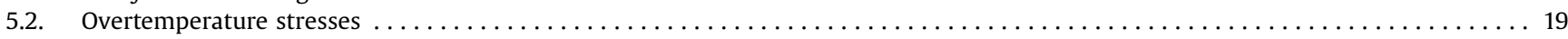

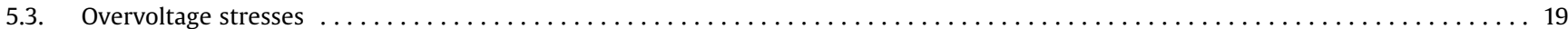

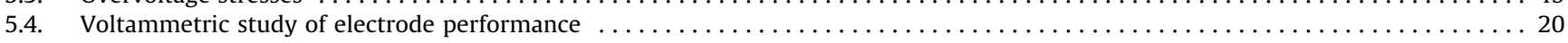

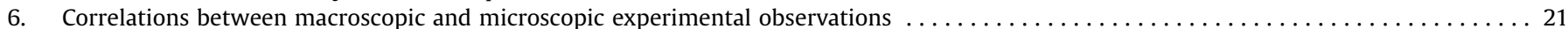

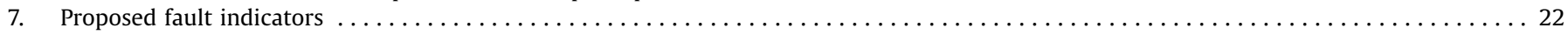

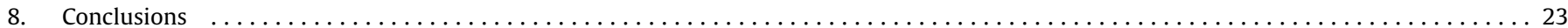

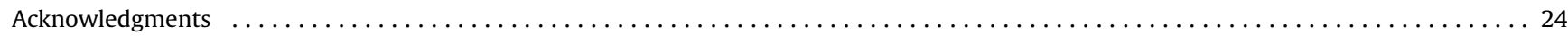

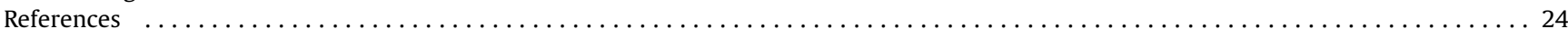

* Corresponding author. Tel.: +41 787209227; fax: +41216934662.

E-mail address: dimitri.torregrossa@epfl.ch (D. Torregrossa). 


\section{Introduction}

Supercapacitors (SCs) are electrical storage devices used in several applications requiring high power densities. The power that can be extracted from a SC depends on its size, state-of-charge (SoC) and on its state-of-health (SoH). This last quantity is extremely important since it directly depends on the equivalent series resistance (ESR) of the SC and on its capacitance $C_{\mathrm{SC}}$ : two parameters that can be easily evaluated.

The literature concerning the supercapacitor $\mathrm{SoH}$ and relevant fault diagnosis is quite limited and can be summarised by the references [1-5]. As discussed in these papers, SC failures appear during ageing causing degradation of their energy storage capabilities and power performances. In several applications the improvement of SC reliability is fundamental and the evaluation of their SoH remains a priority in order to ensure timely maintenance interventions.

In Ref. [1] the SC diagnosis, during operating conditions characterised by the SC temperature $\left(T_{\mathrm{SC}}\right)$ and SC voltage $\left(V_{\mathrm{SC}}\right)$ not exceeding the nominal values, has been obtained by comparing the $V_{\mathrm{SC}}$ slope in charging and discharging phases during ageing test with the corresponding values estimated prior to ageing. The authors of Refs. [3] and [4] focused on the analysis of the changes taking place in the electrochemical properties of generic organic electrolytes (also used in SC) when they are stressed by high temperatures and voltages. References [1-4] have not studied the SC performances when they are stressed with $T_{\mathrm{SC}}$ and $V_{\mathrm{SC}}$ above the nominal values (for $T_{\mathrm{SC}}$ we refer to the electrolyte boiling point generally in the order of $75^{\circ} \mathrm{C}$, and for $V_{\mathrm{SC}}$ to the decomposition voltage generally in the order of $3 \mathrm{~V}$ ). Reference [5] has investigated the performances fading of the SCs with $T_{\mathrm{SC}}$ and $V_{\mathrm{SC}}$ above the nominal values similarly to the current study. However, it is worth making the following observations to better position the research presented in our paper.

i) The SC under test has not cycled during the overtemperature stresses. This is a quite important difference with respect to our work since we have investigated the capability of the SC to deliver high-pulsed current during (and after) overtemperature stresses.

ii) Concerning the power cycling stress, the suggested reference used a current value close to (or below) the rated one. On the contrary, in our power cycling stress, we used currents ranging from the nominal value up to 5 times higher than rated one. In this respect, it is important to underline that for SCs it is difficult to define a maximum rated current in view of their inherent capability to deliver high-power pulsed currents. Therefore, we do think that limiting the investigation to currents below the rated one does not represent a realistic operation condition.

iii) Despite some notable comparable observations that compliment our own conclusions, the post mortem analysis is not as detailed as we have presented in our own paper. We reported seeing cracking in the electrode surface in the over temperature study, and also evidence of arcing in the overvoltage study.

iv) Our study includes a nucler magnetic resonance (NMR) analysis of the electrolyte after overvoltage to ascertain the yellow contaminate.

v) Our analysis of the electrodes via SEM are more detailed and also discuss the crystalline aluminium nanoparticles formed and the polymerisation of the electrolyte.

vi) There is a different method to measure the supercapacitor capacitance during its degradation. Indeed, the above reference has adopted the impedance spectroscopy method whilst we have used a standard SC charge/discharge cycle

vii) The operating temperature ranges investigated in the captioned reference are from the ambient temperature up to $105^{\circ} \mathrm{C}$. In our case, the temperature ranges from ambient up to $130^{\circ} \mathrm{C}$.

As expected, accurate supercapacitor $\mathrm{SoH}$ estimation is based on the exact knowledge of the SC ageing processes. A thorough study of the literature related to the experimental investigation of the SC ageing mechanism has been presented in Refs. [6-11]. In these studies two main types of stressing tests have been considered in order to understand the SC ageing mechanisms: (i) Life Endurance (LE) and (ii) Power Cycling (PC) tests. The former allows for evaluating the effects of $V_{\mathrm{SC}}$ and $T_{\mathrm{SC}}$ on the SC life; the latter analyses the effects of the SC extracted current still on its lifetime. Combined PC and LE tests take into account all the possible ageing factors of SCs. Some authors [6] have also identified a particular phase, called the SC recovering phase, taking place once PC is stopped. In these conditions, a temporary increase of the SC capacitance, $C_{S C}$, is observed. The evident consequence is a change in the $\mathrm{SoH}$ and in the power that can be extracted from the SC. However, in the above-listed references, the physical reasons for the recovering phase are not given. In particular, the effects of $T_{\mathrm{SC}}$ on the duration and on the entity of this recovery phase, along with the conditions to enable this phenomenon, have not been fully investigated. Additionally, the above-listed references have not studied this phenomenon associated to high-pulsed currents delivered by these storage devices.

In this respect, we can summarise the main contributions of this work by the two main items listed below.

1) The first contribution of the paper concerns the fault diagnosis of SCs when used above its nominal $T_{\mathrm{SC}}$ and $V_{\mathrm{SC}}$ operating conditions. The dynamic behaviour of the SC, together with the consequent physical damage after having achieved these extreme operating conditions, has been investigated and analysed. In particular, the three fault indicators are related to the evolution of the following SC parameters: (i) the internal resistance, (ii) capacitance and (iii) decay voltage during relaxation phase. The physical damage relating to these indicators is supported by a post-mortem analysis of SCs using scanning electron microscopy (SEM) and voltammetry to emulate the SC conditions.

2) The second contribution of the paper refers to the identification of a new SC recovering phase taking place during LE towards PC transitions. Furthermore, new sub-phases termed accelerated ageing phases, are studied and reported for the first time. They are defined by a period during which higher $C_{\mathrm{SC}}$ deterioration is observed.

The paper is structured as follows: Section 2 describes the experimental test bench used in this work. Section 3 describes the results related to the over-temperature and overvoltage stresses. The evolution of the above fault indicators is analysed and correlated to possible physical explanations. Section 4 proposes a specific analysis of the $C_{\mathrm{SC}}$ evolution during combined PC and LE tests at three different temperatures in order to highlight the above recovering process and accelerated ageing phases. The description of the phenomena driving these phases is proposed. In Section 5 the post-mortem analysis of some samples of the targeted SCs is given. The results obtained with the scanning electron microscope (SEM), along with the voltammetry of the SC electrode, are in agreement with the analysis of section 2 . Section 6 summarises the link between the fault diagnosis indicators proposed in Section 2 and the microscopic analysis described in Section 4. In Section 7 a fault diagnosis algorithm to distinguish between over-temperature and over-voltage stress is proposed. Section 8 concludes the paper by summarising the main findings. 


\section{Experimental}

\subsection{Targeted SCS}

The electric double layer capacitors used in this investigation were all supplied by the same manufacturer. They had a capacity of $360 \mathrm{~F}$ and operating voltage of $0-2.7 \mathrm{~V}$. It was established from the manufacturers that the capacitor electrolyte is composed of $20-30 \%$ tetraethylammonium tetrafluoroborate $\left(\mathrm{TEA} \mathrm{BF}_{4}\right)$ in acetonitrile. The electrodes comprise porous activated carbon with carbon black as a conductivity modifier possibly bound with a patented $n$-vinyl acetamide polymer binder containing butadiene styrene nanoparticles (note that the binder could not be confirmed due to IP restrictions). This carbon composite was distributed on both sides of an aluminium foil current collector, housed in aluminium casing. All connections were aluminium, and the separator was thin porous paper. The electrolyte conditions were emulated using HPLC grade acetonitrile (Macron Fine Chemicals, United States) with $99.0 \%$ reagent grade $\mathrm{TEABF}_{4}(\mathrm{ABCR}$, Germany). A concentration of $25 \% \mathrm{TEABF}_{4}(1 \mathrm{M})$ was used in the voltammetric studies.

\subsection{Experimental test bench and parameters assessment}

Electrochemical measurements were made using an Autolab PGSTAT 302N (Metrohm, Switzerland) in 3 electrode and 2 electrode arrangements depending on the study. For two-electrode arrangements, the electrodes were of the same geometric area, and were taken from an unused SC. They, therefore, comprised activated carbon, conductivity modifier and binder on the surface of an aluminium backing. Threeelectrode arrangements used an activated carbon working electrode as described above, a platinum wire counter electrode and a $\mathrm{Ag} / \mathrm{AgBF}_{4}$ pseudo reference electrode.

High resolution scanning electron microscopy (HR-SEM) was performed using a Zeiss MERLIN (Carl Zeiss, Germany) instrument with Schottky field emitter. Typical working tension is $1-3.5 \mathrm{keV}$. Standard SEM was performed using a Phillips (FEI) XLF-30 FEG Schottky field-emission microscope operated at beam voltages between 1 and $30 \mathrm{keV}$. Energy-dispersive X-ray (EDX) spectroscopy images were recorded on the latter microscope using a Si detector. The time of acquisition was $120 \mathrm{~s} .{ }^{1} \mathrm{H}$-nuclear magnetic resonance (NMR) spectroscopy was performed using a $400 \mathrm{~Hz}$ Biospin Avance-400 spectrometer (Bruker, US). The sample was prepared by washing the electrode three times in deuterated acetonitrile, retaining each wash, which was then evaporated under nitrogen to concentrate the unknown compound. Viscosity was measured using a SV-1A series viscometer (A and D, Japan) calibrated to pure acetonitrile with the temperature regulated by a circulating water bath.

The power test setup, Fig. 1 illustrates the block scheme of the experimental test bench. It is composed of four main elements: (i) a

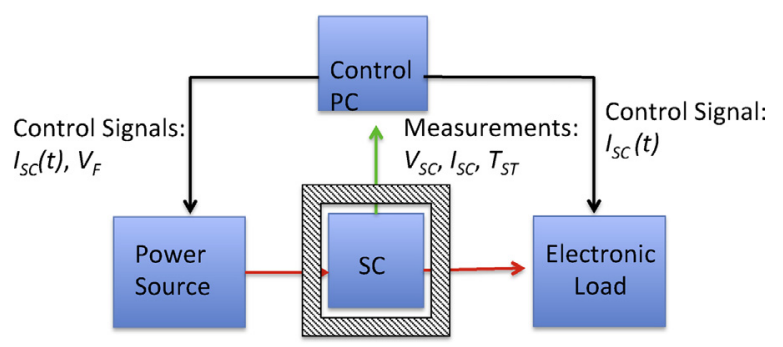

Fig. 1. Block scheme of the experimental test setup. (For interpretation of the references to colour in this figure legend, the reader is referred to the web version of this article.) climatic chamber hosting the targeted SC with operating temperatures in the range $-40^{\circ} \mathrm{C}$ up to $360^{\circ} \mathrm{C}$, in steady state conditions the temperature ripple is of $\pm 0.2^{\circ} \mathrm{C}$; (ii) a power source working in the following $\mathrm{V} / \mathrm{I}$ ranges $0-80 \mathrm{~V}, 0-120 \mathrm{~A}$; (iii) an electronic load working in the following $\mathrm{V} / \mathrm{I}$ ranges $0-80 \mathrm{~V}, 0-200 \mathrm{~A}$; (iv) control PC where a suitable realised software developed using the LabView programming environment is able to perform the monitoring and control the whole system. The electronic load and power supply were characterised by an overall bandwidth from DC to $1 \mathrm{kHz}$. The supercapacitor voltage $\left(V_{\mathrm{SC}}\right)$ was directly sampled using an analog-to-digital converter operating a 16 bits with a maximum sampling frequency of $100 \mathrm{kHz}$ and characterised by a bandwidth from $\mathrm{DC}$ to $100 \mathrm{kHz}(-3 \mathrm{~dB})$ with an overall accuracy of $1.5 \mathrm{mV}$. The supercapacitor current $\left(I_{\mathrm{SC}}\right)$ was measured using a dedicated hall-effect sensor characterised by an overall bandwidth from DC to $100 \mathrm{kHz}(-3 \mathrm{~dB})$. Also for the $I_{\mathrm{SC}}$, the analog output of the current sensor was sampled with a analog-to-digital convert with the same characteristics as the one used for the $V_{\mathrm{SC}}$.

In Fig. 1 the black lines represent the control signals from the control PC towards the power source and the electronic load. During the charging phase the power source receives the set points of the SC charging current and the SC final voltage to be reached at the end of the charge. During the SC discharge the electronic load receives the waveform of the SC discharge current. The red lines of Fig. 1 indicate the transfer of the power (i.e. from the power sources to the SC during the charging phase and from the SC to the electronic load during the discharging phase). The green line indicates the measurement signals: the $V_{\mathrm{SC}}, I_{\mathrm{SC}}$ and $T_{\mathrm{SC}}$ are continuously measured.

The procedure for assessing the SC parameters was structured as follows: (i) the SC was charged with a constant current of $10 \mathrm{~A}$ until it reached a final voltage value of $2.45 \mathrm{~V}$. During this phase the ESR was evaluated by measuring the voltage drop across the SC terminals ${ }^{1}$; (ii) the power generator was turned off and the charge redistribution phenomenon was observed and recorded for $20 \mathrm{~min}$, the value of $V_{\mathrm{SC}}$ at $20 \mathrm{~min}$ (namely $V_{20}$ ) was measured; (iii) the SC was discharged at a constant resistance equal to $0.1 \Omega$ until $V_{\mathrm{SC}}$ reached $100 \mathrm{mV}$. During this last phase the capacitance $C_{\mathrm{SC}}$ was computed as follows:

$C_{\mathrm{SC}}=\frac{\Delta Q_{\mathrm{DISCH}}}{\Delta V_{\mathrm{DISCH}}}$

where $\Delta Q_{\mathrm{DISCH}}$ is the time integral of the current delivered by the SC and $\Delta V_{\text {DISCH }}$ the voltage variation during phase (iii). Deep discharge of the SC until $V_{\mathrm{SC}}=100 \mathrm{mV}$ took around $20 \mathrm{~min}$. The duration of this last time window depends strongly on the value of $V_{\mathrm{SC}}$ required at the end of the deep discharge. The value of $100 \mathrm{mV}$ was chosen as a trade-off between the duration of the test and the evaluation of the SC extracted charge.

Concerning the LE test, the test started once the average temperature of the SC, measured by three thermocouples (on the bottom, up and lateral side of the SC), was equal to the targeted temperature.

For the PC tests, after each discharge the SC was charged at a constant current of $10 \mathrm{~A}$ until it reached a final voltage value $V_{\mathrm{F}}=2.45 \mathrm{~V}$, then the SC was discharged by a current with a pulsed profile. The rest time between two subsequent shots was chosen in the range of a few tens of seconds; the ageing test was stopped each 500 discharges in order to carry out the characterisation test described as above. Further details on the experimental procedure for the SC parameters assessment can be found in Ref. [7].

\footnotetext{
${ }^{1}$ Concerning the ESR, it is defined as the ratio between the variation of $v_{\mathrm{sc}}$ right after the stopping of the SC charging at time $t^{*}$ (i.e. $\Delta v_{\mathrm{sc}}\left(t^{*}\right)$ and the associated charging current $i_{\mathrm{sc}}$.
} 


\section{SCs fault diagnosis for high temperature and high voltage stresses}

This section illustrates the SC fault diagnosis subsequent to high-temperature or high-voltage stresses. Since the diagnosis is based on experimental evidences, in this section, and in the rest of the paper, we make reference to a group of electric double layer SCs of $360 \mathrm{~F}$ described in Section 2.2. In what follows particular attention is given to the SC charge redistribution phase that takes place several hundreds of seconds after the SC open circuit voltage $\left(V_{\mathrm{OC}}\right)$ has been reached. In this respect, the evolution of the SC voltage, henceforth called decay voltage, has been observed for a time window of $20 \mathrm{~min}$. All the findings of this section are supported by the SC post-mortem analysis described in Section 4.

\subsection{Analysis of SC behaviour after high-temperature stresses}

SCs are generally stored at a maximum temperature of $70^{\circ} \mathrm{C}$ and operated at a maximum temperature of $65^{\circ} \mathrm{C}$. In what follows we make reference to a $T_{\mathrm{MAX}}=70^{\circ} \mathrm{C}$ since it represents the extreme SC temperature stress.

If the SC is operated at a nominal temperature of $25^{\circ} \mathrm{C}$, and the $T_{\text {MAX }}$ upper-boundary is never passed, the SC ageing (over a period of 10 years) can be summarised by the following performance fading: (i) $C_{S C}$ loss of $20 \%$; (ii) ESR increase of $100 \%$. For every $10^{\circ} \mathrm{C}$ increase above the nominal SC operating temperature the time period for performance fading decreases by a factor of 2 [8]. It is worth noting that above $T_{\text {MAX }}$ the electrolyte (acetonitrile) is close to its boiling temperature, and within the confines of the sealed capacitor, will have a very high vapor pressure. However, it is reasonable to assume that the SC could be temporarily stressed

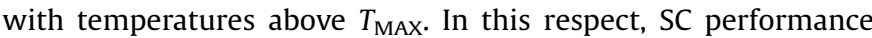
changes have not been comprehensively analysed in the literature thus motivating this work.

A set of specific experiments have been designed; seven over-temperature tests have been subsequently performed to the same SC, respectively at $75^{\circ} \mathrm{C}, 95^{\circ} \mathrm{C}, 115^{\circ} \mathrm{C}, 130^{\circ} \mathrm{C}$ in order to investigate its performance changes relative to those at $25^{\circ} \mathrm{C}$ (note that this value of temperature is declared by the majority of the SC manufactures as the rated one). Additionally, once the $T_{\mathrm{SC}}=130^{\circ} \mathrm{C}$ was reached, the SC was kept at that temperature for $72 \mathrm{~h}$ and its performance evaluated every $24 \mathrm{~h}$. The targeted SC was charged at the maximum voltage of $2.45 \mathrm{~V}$ in each experiment (i.e. well below the maximum SC charging voltage).

The aim of the proposed activity is to investigate the performances fading (in terms of $C_{\mathrm{SC}}$ decrease, ESR increase and decay voltage increase) once an SC is stressed repetitively with an increasing temperature stress. In this way it is possible to detect the condition for which the SC has to be replaced.

Table 1 summarises the measured value of ESR, $C_{\mathrm{SC}}$ and $V_{20}$ during the above over-temperature stress tests. Fig. 2 illustrates the time evolution of the $V_{\mathrm{SC}}$ during the redistribution phases

Table 1

Summary of the SC parameters after consecutive over-temperature stresses.

\begin{tabular}{llll}
\hline \multirow{2}{*}{ Temperature } & \multicolumn{4}{l}{ ESR, $C_{\mathrm{SC}}, V_{20}$ evolution after over-temperature stresses } \\
\cline { 2 - 4 } & ESR $[\mathrm{m} \Omega]$ & $C_{\mathrm{SC}}[\mathrm{F}]$ & $V_{20}(\mathrm{~V})$ \\
\hline $25^{\circ} \mathrm{C}$ & 3.2 & 347 & 2.27 \\
$75^{\circ} \mathrm{C}$ & 3.42 & 351 & 2.17 \\
$95{ }^{\circ} \mathrm{C}$ & 3.54 & 357 & 2.13 \\
$115^{\circ} \mathrm{C}$ & 3.7 & 362 & 1.97 \\
$130^{\circ} \mathrm{C}$ & 3.9 & 369 & 1.92 \\
$130^{\circ} \mathrm{C}-24 \mathrm{~h}$ & 4.9 & 377 & 1.73 \\
$130^{\circ} \mathrm{C}-48 \mathrm{~h}$ & 5.4 & 412 & 1.76 \\
$130^{\circ} \mathrm{C}-72 \mathrm{~h}$ & 6.2 & 422 & 1.76 \\
\hline
\end{tabular}

at the above temperatures. Furthermore, in order to investigate the capability of the SC to deliver high-pulsed currents (i.e. in high-power pulsed applications) during over-temperature stresses, Fig. 3 compares the current delivered by the SC at different temperatures. The profile of the SC extracted current has been a-priori defined in Ref. [7].

As already discussed in Refs. [6-9], temperature is the main source of SC ageing since it directly acts on the micropore structure of the electrodes. The changes provided by over temperature stresses are not reversible. Additionally, at nominal temperature values the main effect is a decrease of $C_{\mathrm{SC}}$. However, temperatures above the nominal one could lead to an important structural modification of the SC electrodes combined with possible changes of the electrolyte's physical properties (e.g. conductivity, viscosity).

By making reference to the available literature on SCs ageing [1-10], and by observing the results shown in Table 1, it is possible to assume that the ageing mechanisms driving performance fading during extreme over-temperature stresses are not the same as those during temperature tests within the nominal range. In particular, by observing Table 1. and Fig. 2 there are some unexpected, interesting results: (i) an expected increase of the ESR (the value can double after just $72 \mathrm{~h}$ of over temperature stress), (ii) an expected increase in the rate of SC voltage decay during short relaxation phases, (iii) an unexpected $C_{\mathrm{SC}}$ increase.

The fast increase of the ESR is due to the normal process leading a LE test being largely accelerated. Notably, there are two main contributions to the ESR value: the resistance related to the electrode-electrolyte surface interface $\left(S_{\mathrm{SC}}\right)$ and the resistance related to the electrolyte itself [12]. High temperature could have two main effects: (i) the production of impurities and (ii) generation of overpressures in the restricted SC volume when near or exceeding the electrolyte boiling point. The two effects potentially modify the electrode micropore structure and indirectly alter (in general a decrease) the $S_{\mathrm{SC}}$ value.

Concerning the SC decay voltage, we previously showed [13] that an increase of the $T_{\mathrm{SC}}$ results in a more pronounced voltage decay. Notably, the decay voltage observed in Fig. 2 is not related to self-discharge originating from a charge-leakage phenomenon but is related to changes in the redistribution of the residual charge phenomenon (see Ref. [13] for further details). Indeed, at higher temperatures the redistribution of charge stored inside the SC is less effective during both charging and relaxation phases. This results in a lower value of SC residual charges $(\Delta Q)$ stored inside

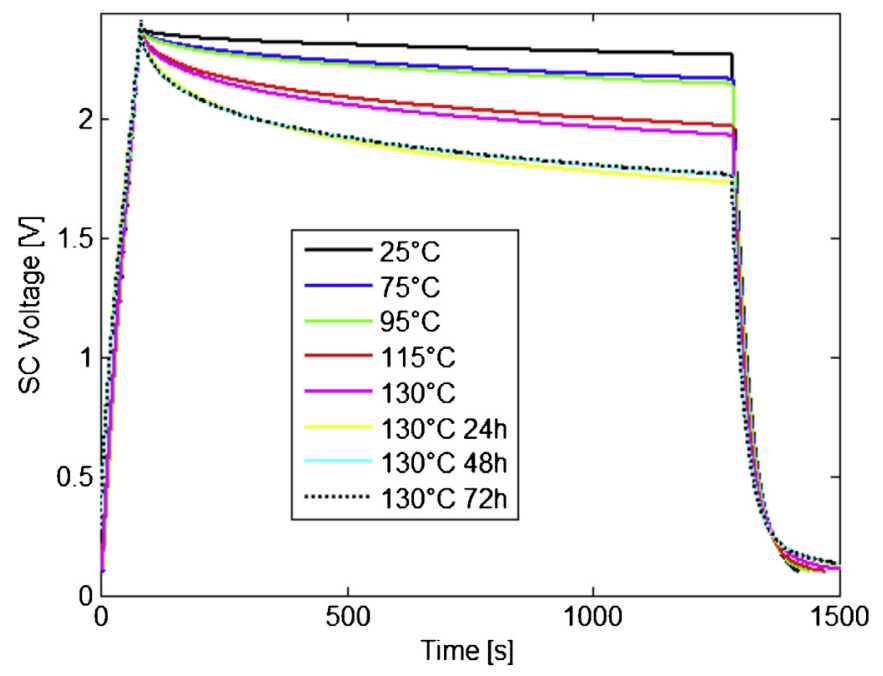

Fig. 2. Evolution of the SC decay voltage, associated to the charge redistribution after consecutive overtemperature stresses. (For interpretation of the references to colour in this figure legend, the reader is referred to the web version of this article.) 


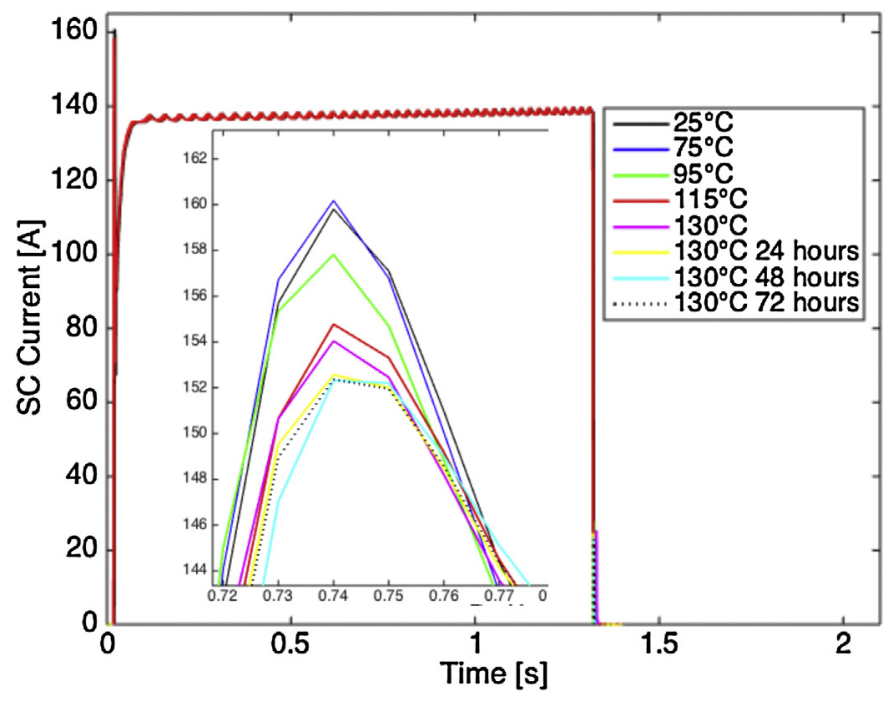

Fig. 3. SC current profiles during high-power pulsed tests after consecutive overtemperature stress (temperature equal to $25^{\circ} \mathrm{C}$ for the parameter assessment subsequent to the first 3 overtemperature stresses).

the SC [13]. Consequently, the decay voltage associated with the following relaxation phase will be more significant.

The $C_{\mathrm{SC}}$ increase is the main unexpected finding since, during normal stress-tests, $C_{\mathrm{SC}}$ fading is expected. In order to give a possible explanation of this finding it is important to underline that at higher temperatures the electrolyte has a higher ionic conductivity (i.e. ion mobility [13,14]). Consequently, the charge extracted by the SC increases resulting in a capacitance increase. Ion mobility increase, along with a mitigated redistribution phenomenon at higher temperatures, could justify the observed $C_{\mathrm{SC}}$ increase.

Concerning the capability of the SC to provide high-pulsed currents (HPC), it can be observed from Fig. 3 that the reduction of the peak current for $T_{\mathrm{SC}}$ up to $95^{\circ} \mathrm{C}$ is negligible. After $72 \mathrm{~h}$ at $130{ }^{\circ} \mathrm{C}$, the peak current that the SC can provide decreases of $6 \%$. Evidently the SC is still able to follow the highly pulsed current profiles in spite of over-temperature stresses. This is mainly due to the fact that the ESR has not considerably increased.

The same set of overtemperature tests have been performed on single cells. In particular, before each overtemperature stress an experimental characterisation of $C_{\mathrm{SC}}$, ESR and $V_{20}$ at $2.45 \mathrm{~V}$ has been performed (Fig. 4). Consequently, in Table 2 the variations of the above parameters have referred in p.u of the values computed at 2.45 V.T. In Table 2 it is possible to observe the same trend evident in Table 1 for subsequent overtemperature stresses. However, the parameters variations are here mitigated with respect to the ones of Table 2 since each targeted SC has been stressed with a single overtemperature (Fig. 5). The results of these two tables can be further interpreted as follows: the ones in Table 1 correspond to an integral set of overtemperature stresses whilst the ones of Table 2 for a single event. The increase of ESR is mitigated of $50 \%$ and the decrease of $V_{20}$ is mitigated of $20 \%$.

\subsection{Analysis of SC behaviour after high-voltage stresses}

SCs are operated to a maximum voltage $V_{\mathrm{MAX}}$ that, in general, is $2.85 \mathrm{~V}$. Similarly to the temperature stress, literature on the subject has identified that an augmentation of $100 \mathrm{mV}$ of $V_{\mathrm{SC}}$ during life endurance tests has a similar effect on the SC performance as the temperature increase of $10^{\circ} \mathrm{C}$ [8]. Even in this case, this empirical rule is valid within the operating voltage range specified by the manufacture (i.e. from $0-V_{\text {MAX }}$ ). SC performance fading, as well as

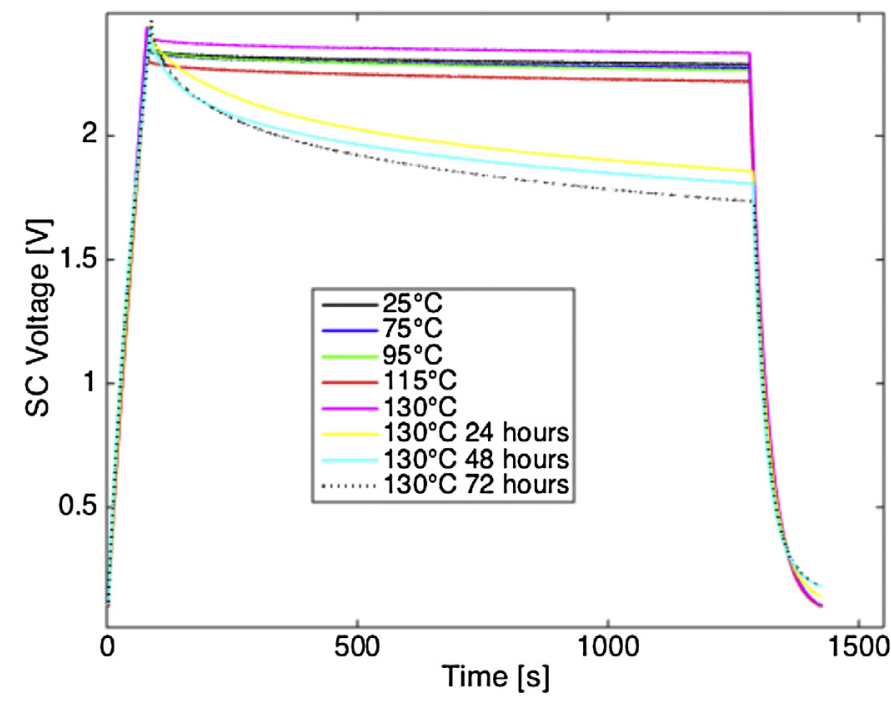

Fig. 4. Evolution of the SC decay voltage, associated to the charge redistribution after single overtemperature stress.

Table 2

Summary of the SC parameters after single over-temperature stresses (p.u. values are referred to the corresponding base quanity measured before the stress).

\begin{tabular}{llll}
\hline \multirow{2}{*}{ Temperature } & \multicolumn{4}{l}{ ESR, $C_{\mathrm{SC}}, V_{20}$ evolution after over-temperature stresses } \\
\cline { 2 - 4 } & $E_{\mathrm{SR}}[\mathrm{m} \Omega]$ & $C_{\mathrm{SC}}[\mathrm{F}]$ & $V_{20}$ \\
\hline $75^{\circ} \mathrm{C}$ & 1.03 p.u. & 1.01 p.u. & 1 p.u. \\
$95^{\circ} \mathrm{C}$ & 1.06 p.u. & 1.03 p.u. & 1 p.u. \\
$115^{\circ} \mathrm{C}$ & 1.08 p.u. & 1.04 p.u. & 0.97 p.u. \\
$130^{\circ} \mathrm{C}$ & 1.09 p.u. & 1.10 p.u. & 0.9 p.u. \\
$130^{\circ} \mathrm{C}-24 \mathrm{~h}$ & 1.1 p.u. & 1.21 p.u. & 0.87 p.u. \\
$130^{\circ} \mathrm{C}-48 \mathrm{~h}$ & 1.48 p.u. & 1.22 p.u. & 0.83 p.u. \\
$130^{\circ} \mathrm{C}-72 \mathrm{~h}$ & 1.59 p.u. & 1.22 p.u. & 0.75 p.u. \\
\hline
\end{tabular}

SC dynamic behaviour when the $V_{\mathrm{SC}}$ passes the upper limit, has not been comprehensively investigated in the available literature. Since an overvoltage can be unintentionally yet easily applied to SCs the findings presented in this section aim to provide an associated performance evaluation.

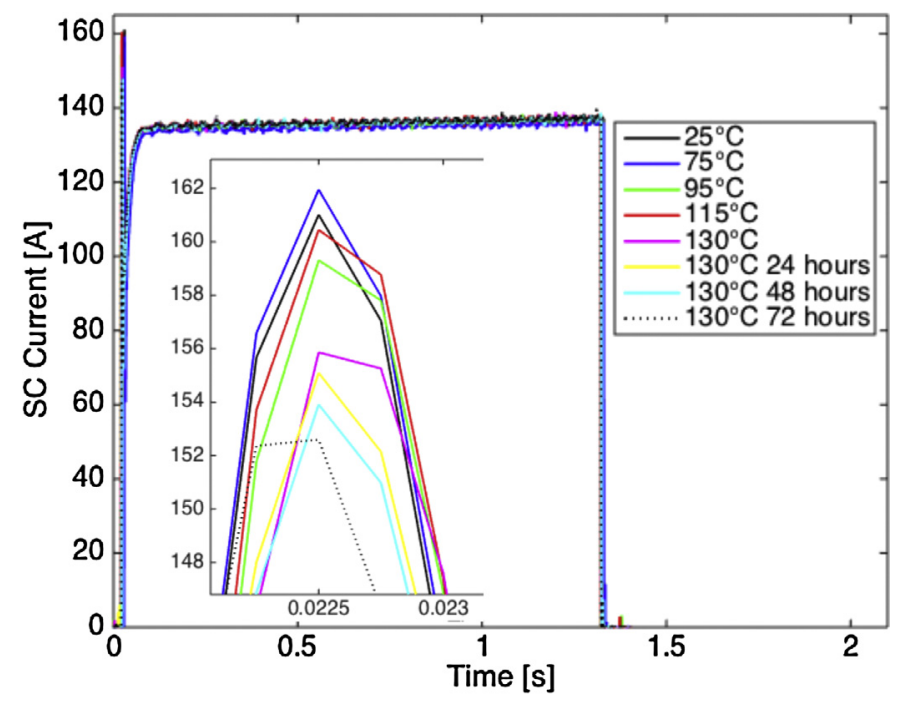

Fig. 5. SC current profiles during high-power pulsed tests after single overtemperature stress (temperature equal to $25{ }^{\circ} \mathrm{C}$ for the parameter assessment subsequent to the first 3 overtemperature stresses). 
Table 3

Summary of the SC parameters after consecutive over-voltage stresses.

\begin{tabular}{llll}
\hline \multirow{2}{*}{ Applied voltage $(\mathrm{V})$} & \multicolumn{3}{l}{ ESR, $C_{\mathrm{SC}}, V_{20}$ evolution after over-temperature stresses } \\
\cline { 2 - 4 } & $\mathrm{ESR}[\mathrm{m} \Omega]$ & $C_{\mathrm{SC}}[\mathrm{F}]$ & $V_{20}(\mathrm{~V})$ \\
\hline 2.45 & 2.81 & 359.5 & 2.3 \\
3 & 2.81 & 370 & 2.3 \\
3.25 & 2.81 & 400 & 2.27 \\
3.5 & 4.31 & 338 & 2.19 \\
3.75 & 6.2 & 296 & 1.87 \\
4 & 31 & 285 & 1.62 \\
\hline
\end{tabular}

In this respect, five over-voltage tests have been subsequently performed to the same SC, respectively at $3 \mathrm{~V}, 3.25 \mathrm{~V}, 3.5 \mathrm{~V}, 3.75 \mathrm{~V}$ have and $4 \mathrm{~V}$ belonging to the same set used for the over temperature stresses. These tests have been used to investigate the SC performance changes compared to those when it was charged at $2.45 \mathrm{~V}$ (this value is lower than the rated one, namely $2.7 \mathrm{~V}$. please, note that this value of voltage is quite common for the majority of the SCs). ${ }^{2}$ The targeted SCs have been placed in a climatic chamber at $25^{\circ} \mathrm{C}$. It is important to underline that, once the voltage reached the predefined value, the SC has been cycled for few minutes with the same current profile used during the over-temperature stress-tests. During the tests at $3.75 \mathrm{~V}$ and $4 \mathrm{~V}$ the $T_{\mathrm{SC}}$ was observed to temporarily reach $60^{\circ} \mathrm{C}$. The temperature was equal to $25^{\circ} \mathrm{C}$ during the parameters assessment following each overvoltage stress.

The experimental findings are summarised in Table 3 specifically the ESR, $C_{\mathrm{SC}}$ and $V_{20}$ values after the different over-voltage tests. Fig. 6 illustrates the time evolution of the $V_{\mathrm{SC}}$ during long redistribution phases subsequent to each overvoltage test. Fig. 7 illustrates the current profile delivered by the targeted SC after each test.

The over-voltage stressed SCs displayed the following behaviors (i) an unexpected large increase of the ESR (this parameter can be 10 times bigger than the value measured at the nominal voltage), (ii) an unexpected increase of the decay voltage during short relaxation phases, (iii) an expected $C_{\mathrm{SC}}$ decrease following an unexpected $C_{\mathrm{SC}}$ increase. These observations suggest that the ageing mechanism driving performance fading during the proposed extreme over-voltage stress tests are not the same as the ones operating during voltage stress-tests within the nominal range of $V_{\mathrm{SC}}$.

Concerning the fast increase of the ESR, it is important to remember that during the cycling phase the $T_{\mathrm{SC}}$ also increased. Apparently, there is no reason for this $T_{\mathrm{SC}}$ augmentation but its explanation, furnished in this paragraph, could support the ESR evolution. Previous work [15] has identified the possibility of microcracking on the electrode surfaces during SC cycling. These microcracks could modify the electrode-electrolyte interface, namely the $S_{\mathrm{Sc}}$. Additionally, once the SC is cycled, these microcracks could cause short-circuiting and therefore associated heat generation. Short circuiting would indeed briefly and sharply raise the temperature of the SC followed by a dissipation of that energy through the volume of the $\mathrm{SC}$ to raise the overall $T_{\mathrm{SC}}$. This temperature increase, probably due to these short-circuits, intensifies all the ageing mechanisms previously described for the over-temperature stress-tests. Additionally, it is likely that the

\footnotetext{
2 The charge redistribution taking place in SC when charged with a final voltage of $2.7 \mathrm{~V}$ is quite important. This involves an asymptotic SC open-circuit voltage that, in average, is in the range of $2.45 \mathrm{~V}$. If the $\mathrm{SC}$ is charged with a lower final voltage the asymptotic SC open-circuit voltage is much closer to the final charging one (for a final charging voltage of $2.45 \mathrm{~V}$ we obtain an open circuit voltage of $2.40 \mathrm{~V}$ ). The consequence is that, normally, SCs are operated with final charging voltages lower than the maximum ones. The value of $2.45 \mathrm{~V}$ has been selected as a representative value.
}

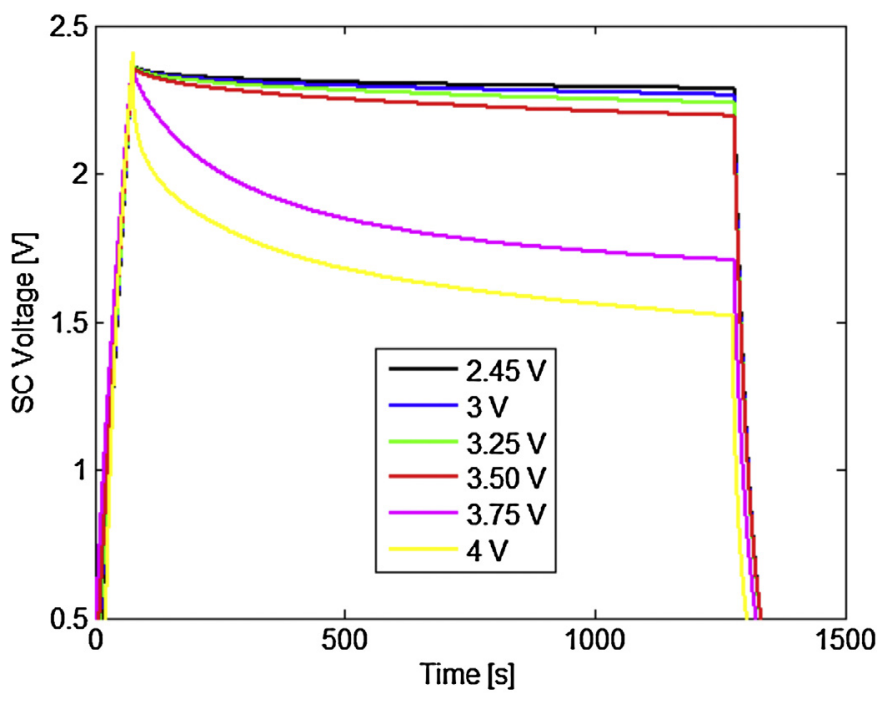

Fig. 6. Evolution of the SC decay voltage, associated to the charge redistribution after consecutive overvoltage stresses.

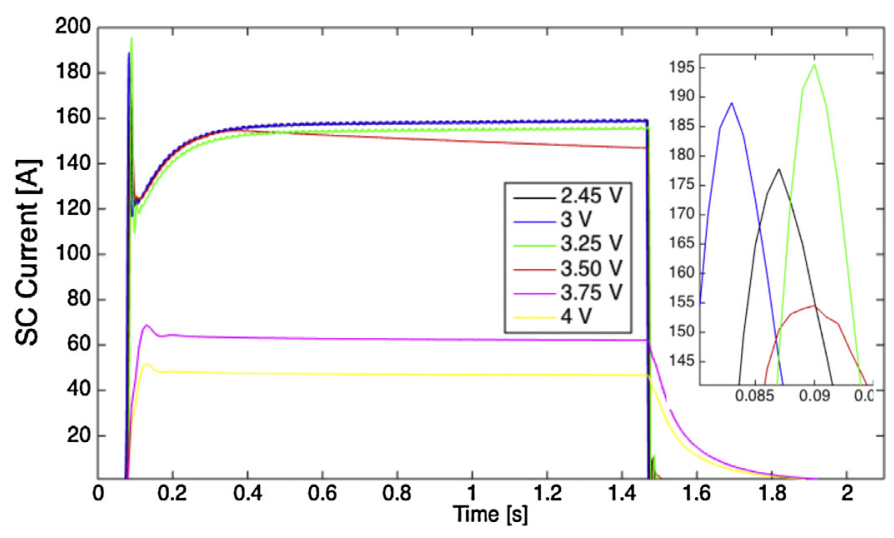

Fig. 7. SC current profiles during high-power pulsed tests after consecutive overtevoltage stress (temperature equal to $25^{\circ} \mathrm{C}$ for the parameter assessment phases).

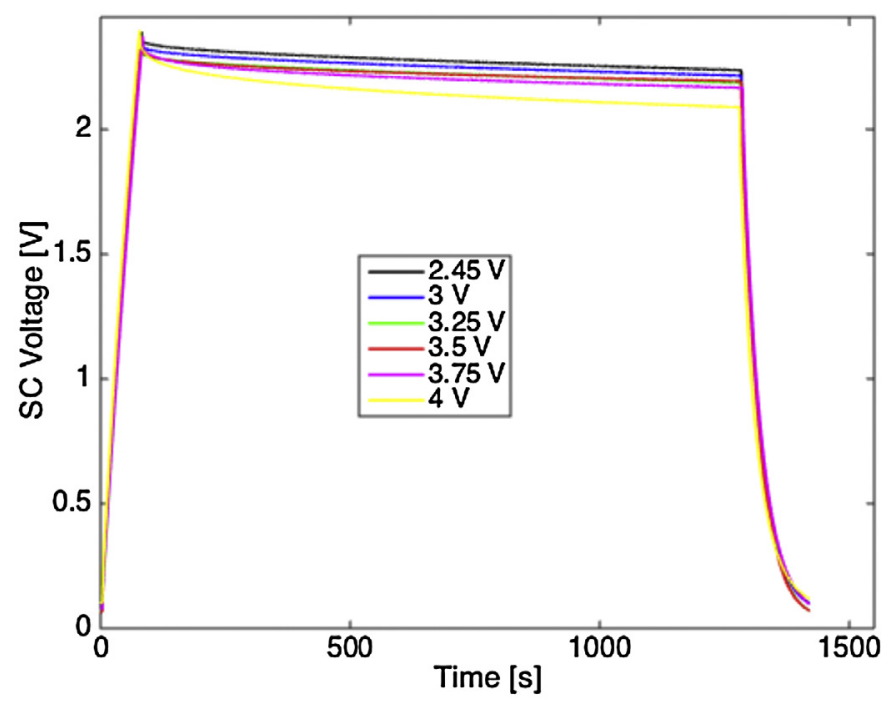

Fig. 8. Evolution of the SC decay voltage, associated to the charge redistribution after single overvoltage stresses. 


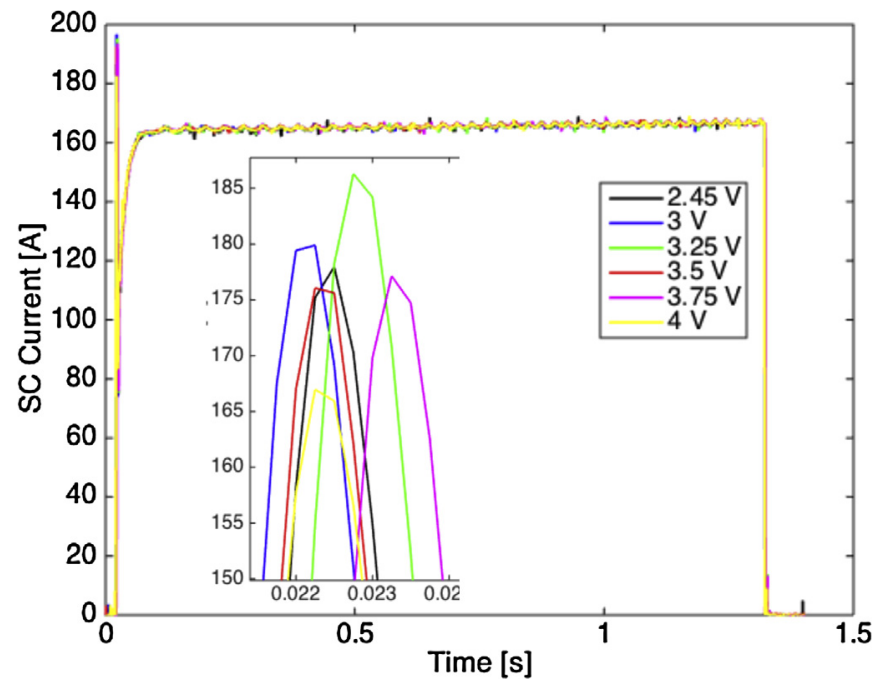

Fig. 9. SC current profiles during high-power pulsed tests after single overvoltage stress (temperature equal to $25^{\circ} \mathrm{C}$ for the parameter assessment phases).

decomposition voltage of the electrolyte was attained during the higher overvoltage stresses creating further impurities to decrease the $S_{\mathrm{SC}}$ and also changing the electrolyte conductivity and viscosity.

As already explained in the previous subsection, the SC decay voltage is related to changes in the redistribution of the residual charge. For the same reasons given in the previous subsection, the redistribution phenomenon is mitigated by the consequent increase of the temperature. Furthermore, decomposition of the electrolyte would modify charge transfer by altering the electrolyte viscosity, ionic strength, conductivity, and introducing redox active impurities. These reasons could explain the extreme intensification of the decay voltage trend, particularly after $3.5 \mathrm{~V}$.

During the first tests the $C_{\mathrm{SC}}$ value is seen to increase, then rapidly decline at voltages above $3.25 \mathrm{~V}$. Again, the overvoltage introduces over-temperature effects to the $C_{\mathrm{SC}}$ already discussed in the previous sub-section. However, once the $V_{\mathrm{SC}}$ has reached a certain value, very fast fading is observed, specifically $20 \%$ in few hours of cycling at over-voltage. This result may be explained by the decomposition of the electrolyte, modifying its properties and reducing charge penetration into the electrode pores. Moreover, the production of impurities, electrolyte decomposition and possible microcracking, could considerably reduce the $S_{\mathrm{Sc}}$. This indirectly causes significant fading of the $C_{\mathrm{sc}}$.

In Fig. 7 it can be observed that for $V_{S C}$ values below $3.50 \mathrm{~V}$ the SC high-pulsed power capability does not change. Then, once possible irreversible modifications of the electrolyte and/or electrode take place, the SC is not able to deliver high current values. However, it can still deliver current values close to the nominal one.

The same set of overvoltage tests have been performed on single cells. In particular, before each overvoltage stress an experimental characterisation of $C_{\mathrm{SC}}$, ESR and $\mathrm{V}_{20}$ at $2.45 \mathrm{~V}$ has

\section{Table 4}

Summary of the SC parameters during single over-voltage tests (p.u. values are referred to the corresponding base quantity measured before the stress).

\begin{tabular}{llll}
\hline \multirow{2}{*}{ Applied voltage (V) } & \multicolumn{3}{l}{$E_{\mathrm{SR}}$ and $C_{\mathrm{SC}}$ evolution during over-voltage tests } \\
\cline { 2 - 4 } & $E_{\mathrm{SR}}[\mathrm{m} \Omega]$ & $C_{\mathrm{SC}}[\mathrm{F}]$ & $V_{20}$ \\
\hline 3 & 1 p.u. & 1.02 p.u. & 1 p.u. \\
3.25 & 1 p.u. & 1.05 p.u. & 0.98 p.u. \\
3.5 & 1.06 p.u. & 1.07 p.u. & 0.97 p.u. \\
3.75 & 1.11 p.u. & 0.93 pu. & 0.95 p.u. \\
4 & 1.41 p.u. & 0.88 pu. & 0.91 p.u. \\
\hline
\end{tabular}

been performed. Consequently, in Table 4 the variations of the above parameters have referred in p.u of the values computed at $2.45 \mathrm{~V}$. In Table 4 it is possible to observe the same trend evident in Table 3 for subsequent overvoltage stresses. However, the parameters variations are here mitigated with respect to the ones of Table 3 since each targeted SC has been stressed with a single overvoltage. The results of these two tables can be further interpreted as follows: the ones in Table 4 corresponds to an integral set of overvoltage stresses whilst the ones of Table 3 for a single event. The increase of ESR is mitigated from 30\% up to $900 \%$ and the decrease of $V_{20}$ is mitigated of $15 \%$. The main difference is associated to the capability of the tartegeted SC to still deliver high pulsed current event after the application of a $4 \mathrm{~V}$ voltage.

\section{Ageing and recovering process analysis}

Table 5 illustrates the evolution of the $C_{\mathrm{SC}}$ during a combined LE and PC test at three different temperatures, $25^{\circ} \mathrm{C}, 35^{\circ} \mathrm{C}$ and $45^{\circ} \mathrm{C}$, respectively. The PC and LE ageing tests have been briefly described in Section 2.2. The structure of the whole ageing test is as follows: the first part is the initial combined LE and PC test (time window of $24 \mathrm{~h}$ ), the second is a single LE test (time window of $12 \mathrm{~h}$ ), a third is a combined LE and PC test (time window of $136 \mathrm{~h}$ ), the fourth a single LE test (time window of $27 \mathrm{~h}$ ), the fifth a combined LE and PC test (time window of $109 \mathrm{~h}$ ), the sixth a single LE test (time window of $27 \mathrm{~h}$ ), and the last one is a combined LE and PC test (time window of $45 \mathrm{~h}$ ). After each PC stopping and restart, it is possible to make the specific observations contained in the rest of this section.

The following acronyms are used in Table 4 to clearly identify the different phases observed: normal ageing phase (NA), accelerated phase (AP), recovering phase (RP). At each transition the increase or decrease of $C_{\mathrm{SC}}$ is evaluated and referred to the last value of the previous ageing phase (LE or combined PC and LE test). From Table 5 two main phases may be identified, accelerated ageing phase and recovering phase for which the duration and the associated $C_{\mathrm{SC}}$ evolution depend strongly on the $T_{\mathrm{SC}}$ value.

From Table 5 it is possible to draw the following conclusions: (i) the accelerated ageing phase is detected at any transition of PC test to LE test; (ii) the duration of the accelerated ageing phase seems to be independent of the temperature but the associated $C_{\mathrm{SC}}$ fading strongly depends on the $T_{\mathrm{SC}}$; (iii) the recovering phase takes place during the transition from LE to PC test; (iv) the condition for which the recovering phase takes place along with its duration and associated variations of $C_{\mathrm{SC}}$ depends strongly on the $T_{\mathrm{SC}}$ value and on the duration of the previous LE test.

During the LE test and the combined PC and LE test the $C_{\mathrm{SC}}$ has been recorded every $3 \mathrm{~h}$. The procedure used to evaluate the $C_{\mathrm{SC}}$ has been described in Section 2.2. It is important to note that the observed recovering phase is associated with the utilisation of the SC with high-pulsed current extraction (10 times higher than the nominal value for $1 \mathrm{~s}$ ). To the best of our knowledge there is no available literature on ageing and recovering processes associated to this particular use of the electrochemical double layer SC.

Fig. 10 illustrates the time evolution of the CSC during the combined PC and LE test depicted in Table 5. It is important to underline that the targeted SCs have been placed into a climatic chamber at the various temperatures of $25^{\circ} \mathrm{C}, 35^{\circ} \mathrm{C}, 45^{\circ} \mathrm{C}$ and temporary overtemperatures associated to the PC are within $1.5^{\circ} \mathrm{C}$. For this reason, we have not included in this figure the SC temperature trends.

\subsection{Accelerated ageing phenomenom}

When the SC is aged with a combined PC and LE test the $C_{\mathrm{SC}}$ decreases following quasi-exponential fading [7-10]. The main 
Table 5

Summary of the $C_{\mathrm{SC}}$ evolution during the combined PC and LE tests.

\begin{tabular}{|c|c|c|c|}
\hline \multirow[t]{2}{*}{ Type of test } & \multicolumn{3}{|l|}{$C_{\mathrm{SC}}$ evolution $[\mathrm{F}]$} \\
\hline & $T_{\mathrm{SC}} 25^{\circ} \mathrm{C}$ & $T_{\mathrm{SC}} 35^{\circ} \mathrm{C}$ & $T_{\mathrm{SC}} 45^{\circ} \mathrm{C}$ \\
\hline $3 \mathrm{~h}$ of combined PC and LE & 354.5 & 353 & 349.5 \\
\hline $21 \mathrm{~h}$ of combined PC and LE & 346.1 (NA) & 345.4 (NA) & $341.5(\mathrm{NA})$ \\
\hline $3 \mathrm{~h}$ of LE & $344.6(\mathrm{AP}-0.45 \%)$ & $344(\mathrm{AP}-0.4 \%)$ & $339.6(\mathrm{AP}-0.57 \%)$ \\
\hline $9 \mathrm{~h}$ of LE & $342.9(\mathrm{AP}-0.95 \%)$ & $340.9(\mathrm{AP}-1.3 \%)$ & $336.4(\mathrm{~A}-1.5 \%)$ \\
\hline $3 \mathrm{~h}$ of combined PC and LE & $346.7(\mathrm{RP}+1.21 \%)$ & $344.2(\mathrm{RP}+0.9 \%)$ & $338.7(\mathrm{RP}+0.7 \%)$ \\
\hline $3 \mathrm{~h}$ of combined PC and LE & $347.3(\mathrm{RP}+1.3 \%)$ & $343.6(\mathrm{NA}+0.7 \%)$ & 338. (NA+0.45\%) \\
\hline $130 \mathrm{~h}$ of combined PC and LE & $340.6(\mathrm{NA}-0.65 \%)$ & $338.4(\mathrm{NA}-0.7 \%)$ & $330.4(\mathrm{NA}-1.75 \%)$ \\
\hline $3 \mathrm{~h}$ of LE & $339.4(\mathrm{AP}-0.3 \%)$ & $335.8(\mathrm{AP}-0.7 \%)$ & $327.3(\mathrm{AP}-0.95 \%)$ \\
\hline $12 \mathrm{~h}$ of LE & $338(\mathrm{AP}-0.75 \%)$ & $334.7(\mathrm{AP}-1.1 \%)$ & $326.3(\mathrm{AP}-1.2 \%)$ \\
\hline $3 \mathrm{~h}$ of combined PC and LE & $339.7(\mathrm{RP}+0.5 \%)$ & $337.7(\mathrm{RP}+0.9 \%)$ & $329.2(\mathrm{RP}+0.9 \%)$ \\
\hline $6 \mathrm{~h}$ of combined PC and LE & $340.7(\mathrm{RP}+0.8 \%)$ & $337.7(\mathrm{NA}+0.9 \%)$ & $329.3(\mathrm{NA}+0.93 \%)$ \\
\hline $100 \mathrm{~h}$ of combined PC and LE & $338.2(\mathrm{NA}-0.04 \%)$ & $334.6(\mathrm{NA}-0.4 \%)$ & $327.9(\mathrm{NA}-0.5 \%)$ \\
\hline $3 \mathrm{~h}$ of LE & $336.3(\mathrm{AP}-0.5 \%)$ & $333.1(\mathrm{AP}-0.4 \%)$ & $325(\mathrm{AP}-0.8 \%)$ \\
\hline $24 \mathrm{~h}$ of LE & $335.8(\mathrm{AP}-0.7 \%)$ & $331(\mathrm{AP}-1 \%)$ & $323.9(\mathrm{AP}-1.2 \%)$ \\
\hline $3 \mathrm{~h}$ of combined PC and LE PC & $337.2(\mathrm{RP}+0.4 \%)$ & $333.9(\mathrm{RP}+0.9 \%)$ & $324.2(\mathrm{RP}+0.1 \%)$ \\
\hline $6 \mathrm{~h}$ of combined PC and LE & $338(\mathrm{RP}+0.7 \%)$ & $334.2(\mathrm{RP}+0.95 \%)$ & $326.6(\mathrm{RP}+0.8 \%)$ \\
\hline $36 \mathrm{~h}$ of combined PC and LE & $337.9(\mathrm{RP}+0.7 \%)$ & $334(\mathrm{NA}+0.9 \%)$ & $325.8(\mathrm{NA}+0.5 \%)$ \\
\hline
\end{tabular}

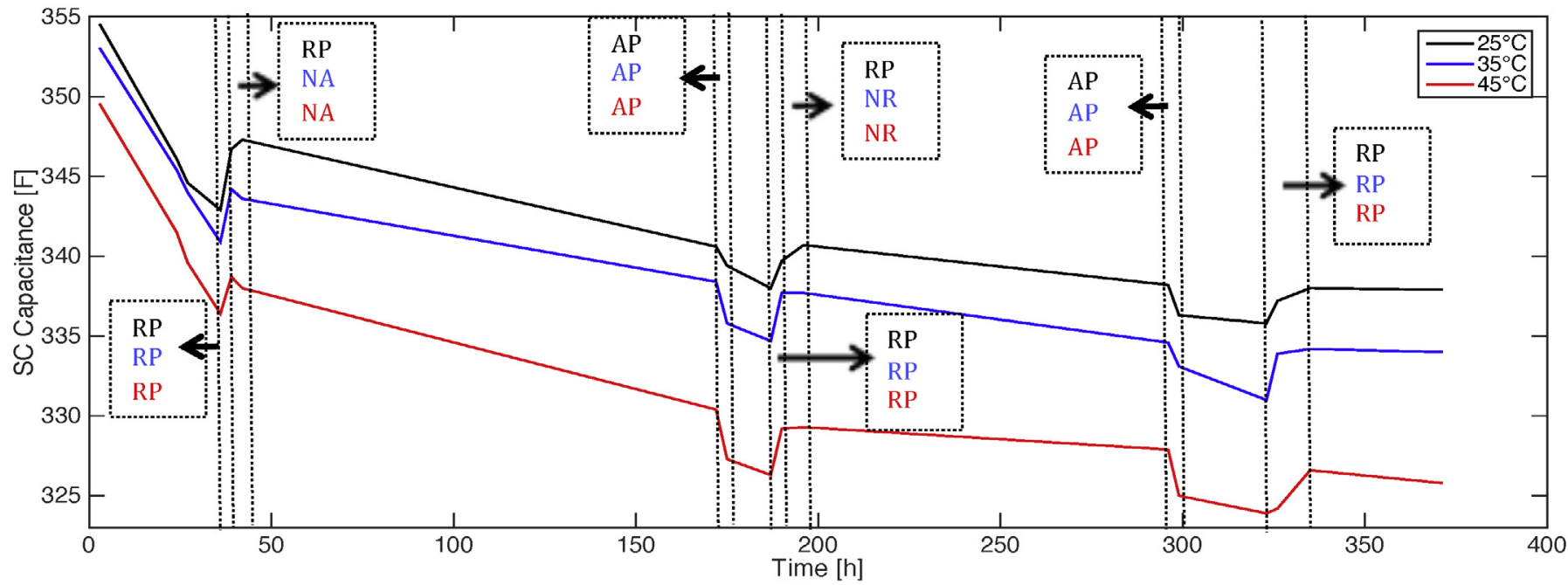

Fig. 10. Evolution of CSC during the combined PC and LE test. The RP and AP are highlighted.

ageing factor, if the $T_{\mathrm{SC}}$ is around $25^{\circ} \mathrm{C}$, is the current extraction, involving an increase of the $T_{\mathrm{SC}}$ and consequent exfoliation of the carbon electrode surface blocking some macro-pores. The structure of the electrode consists of elementary crystallites of activated carbon with a large number of interstices between them. The interstices tend to be filled with carbon residues as conductivity modifier that block the pore entrances during a LE [15]. This latter effect reduces the surface between electrode and electrolyte $\left(S_{\mathrm{SC}}\right)$ and consequently the charge transfer from electrolyte to electrode. It is important to note that some benefits may be associated to higher internal SC temperatures caused by the current extraction: (i) a lower electrolyte viscosity increasing the charge penetration of the electrolyte into the pores; (ii) higher electrolyte conductivity and consequently a higher amount of charge transfer between electrode and electrolyte. Additionally current extraction, especially if the current is pulsed, removes some of the impurities depositing on the above interstices (the quantity of these impurities increases with the temperature). Once the PC is stopped, these above benefits are no longer present, and consequently there is an acceleration of the ageing process, namely a more significant and faster $C_{\mathrm{SC}}$ decrease. The decrease is more important for the SC that has been previously aged at higher temperature (see in Table 5 the row associated to the beginning of each LE test). This happens because the higher temperature involves an increase in the production of impurities and further degradation of the electrolyte (discussed below). One may assume that the $I_{\mathrm{SC}}$ is a mitigating-ageing factor countering the $T_{\mathrm{SC}}$ ageing factor during combined LE and PC tests.

\subsection{Regenerative phenomenom}

During the LE tests, the $C_{\mathrm{SC}}$ follows a quasi-exponential fading vs. time that has been observed in the literature previously [7-10]. The evolution of the $C_{\mathrm{SC}}$ parameter following the transition from a LE test to a combined PC and LE test requires specific analysis. Three different $C_{\mathrm{SC}}$ behaviours can be identified at the beginning of the PC restart, with duration and associated $C_{\mathrm{SC}}$ changes depending strongly on the $T_{\mathrm{SC}}$. The first behaviour identified shows an increase in $C_{\mathrm{SC}}$ which is maintained for a certain period of time, before returning to age following the classical capacitance evolution [7-10]. The second behaviour observed is an increase in $C_{\mathrm{SC}}$ for a certain time duration, after which a normal decrease restarts. The third behaviour is that the $C_{\mathrm{SC}}$ increases then suddenly decreases normally. 
The occurrence of one of the above behaviours depends on the $T_{\mathrm{SC}}$ value and on the duration of the previous LE test. Detailed descriptions of these possibilities are classified for each $T_{\mathrm{SC}}$ used, as follows.

i) If the $T_{\mathrm{SC}}$ is equal to $25^{\circ} \mathrm{C}$ (the nominal temperature specified by the manufacture) once PC resumes, the first sub-phase associated to the recovering phenomena always takes place: the $C_{\mathrm{SC}}$ starts to increase until it reaches a maximum value. During this specific phase the extraction of current partially removes some of the impurities that filled the interstices in the previous phase (the LE test). Additionally, the extraction of current involves an increase of SC temperature due to the internal Joule losses. This triggers the decrease of viscosity and the increase of the electrolyte conductivity. The result is greater penetration of the electrolyte into the pores of the electrode surface and consequently a higher amount of charge transfer. It is important to note that the current extraction, especially because it has the time evolution characteristic of a pulsed current, is causing micro-cracking in the electrode which modifies the porosity of the electrode [15]. The production of these impurities mixed with the electrolyte reduces the electrolyte conductivity as well. It is reasonable to assume that at the beginning of the PC restart there are two processes:
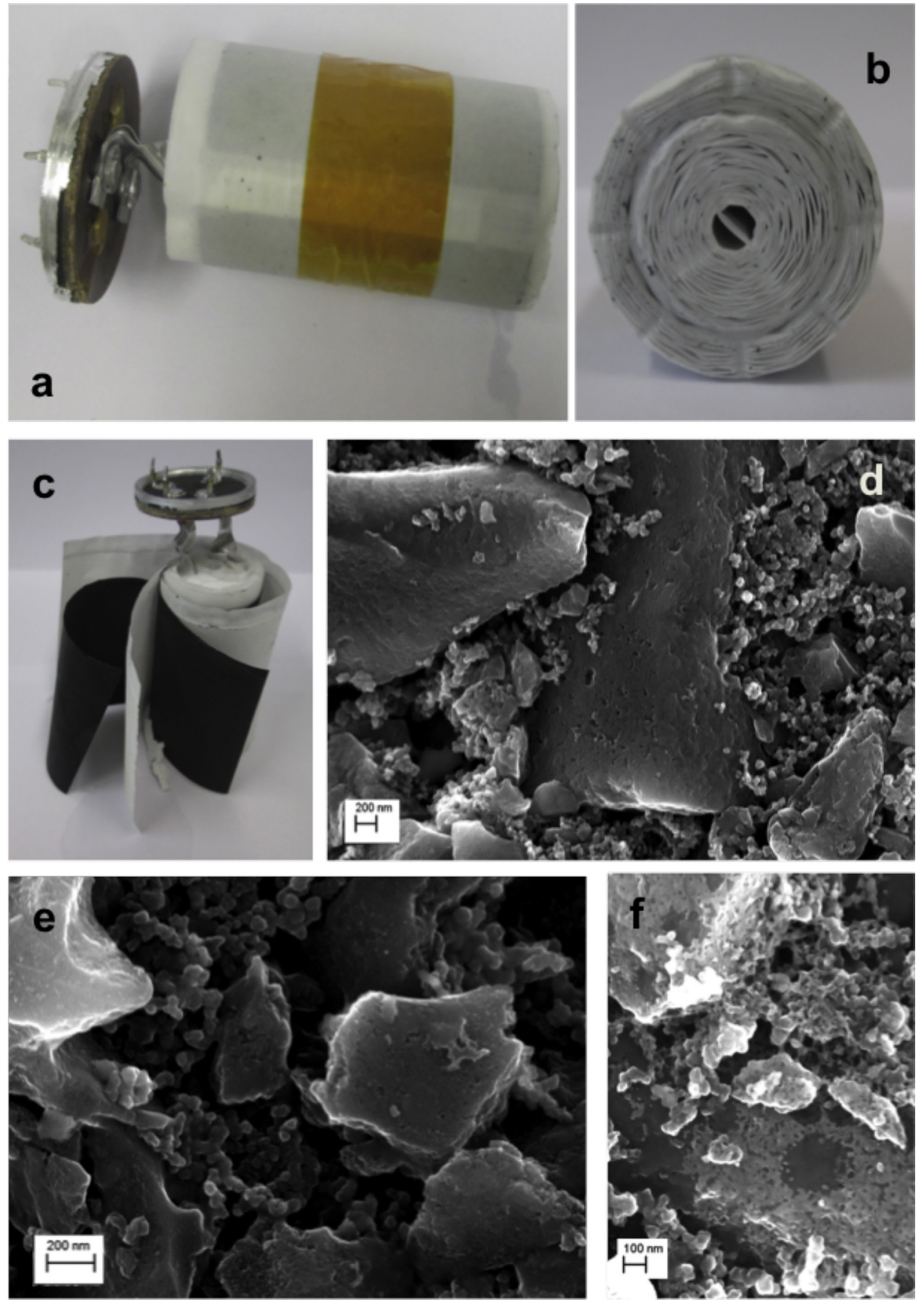

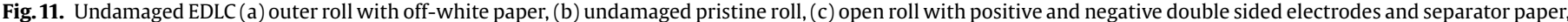

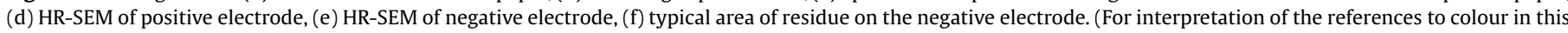
figure legend, the reader is referred to the web version of this article.) 
one involving an ageing phenomena associated to current extraction, another one involving a regenerative phenomena associated to the viscosity and electrolyte conductivity changes. During the first part of this phase $\left(6 \mathrm{~h}\right.$ at $25^{\circ} \mathrm{C}$ in Table 5), the regenerative phenomenon is stronger than the ageing one. Once the $C_{\mathrm{SC}}$ reaches the maximum value, it means that the two processes are perfectly compensated. From this point any further extraction of current is no longer compensated by the above benefit causing a reduction of the $C_{\mathrm{SC}}$ value. The $C_{\mathrm{SC}}$ evolution follows the characteristic fading of a PC test.

At $25^{\circ} \mathrm{C}$, the increase of $C_{\mathrm{SC}}$ evaluated at each transition from LE to combined PC and LE test is decreasing: $+1.21 \%$ at the first transition, $+0.5 \%$ and $0.4 \%$ in the last one. It is reasonable to justify this finding by the fact that the benefit associated to the current extraction becomes lower. In fact the SC SoH at the last transition is lower than the one evaluated at the beginning. The $\mathrm{SoH}$ is mainly related to the filled micropores and the electrolyte properties. At the last transition the benefits associated with PC are relatively less important than those at the beginning of the test (at the beginning of the test the electrolyte has not deteriorated and few micropores are filled with impurities). The duration of the recovering sub-phase is still the same at each transition since it is related to an increase in the temperature (increase of electrolyte conductivity and decrease of viscosity) associated to the current extraction. The increase in temperature is practically the same since the heat capacity of the electrolyte is not significantly changed and the current profile used during the PC test is always the same.

ii) If the $T_{\mathrm{SC}}$ is equal to $35^{\circ} \mathrm{C}$, once $\mathrm{PC}$ is restarted, the $C_{\mathrm{SC}}$ evolution depends on the duration of the previous LE test. As shown in Table 5 during the first transition the recovering phase holds for just 3 hours (the previous LE test held for $12 \mathrm{~h}$ ); during the second transition there is a recovering phase perfectly compensating the main ageing process (the previous LE test held for $15 \mathrm{~h}$ ); during the third transition there is a recovering phase with an associated $C_{\mathrm{SC}}$ increase (the previous LE test held for $27 \mathrm{~h}$ ).
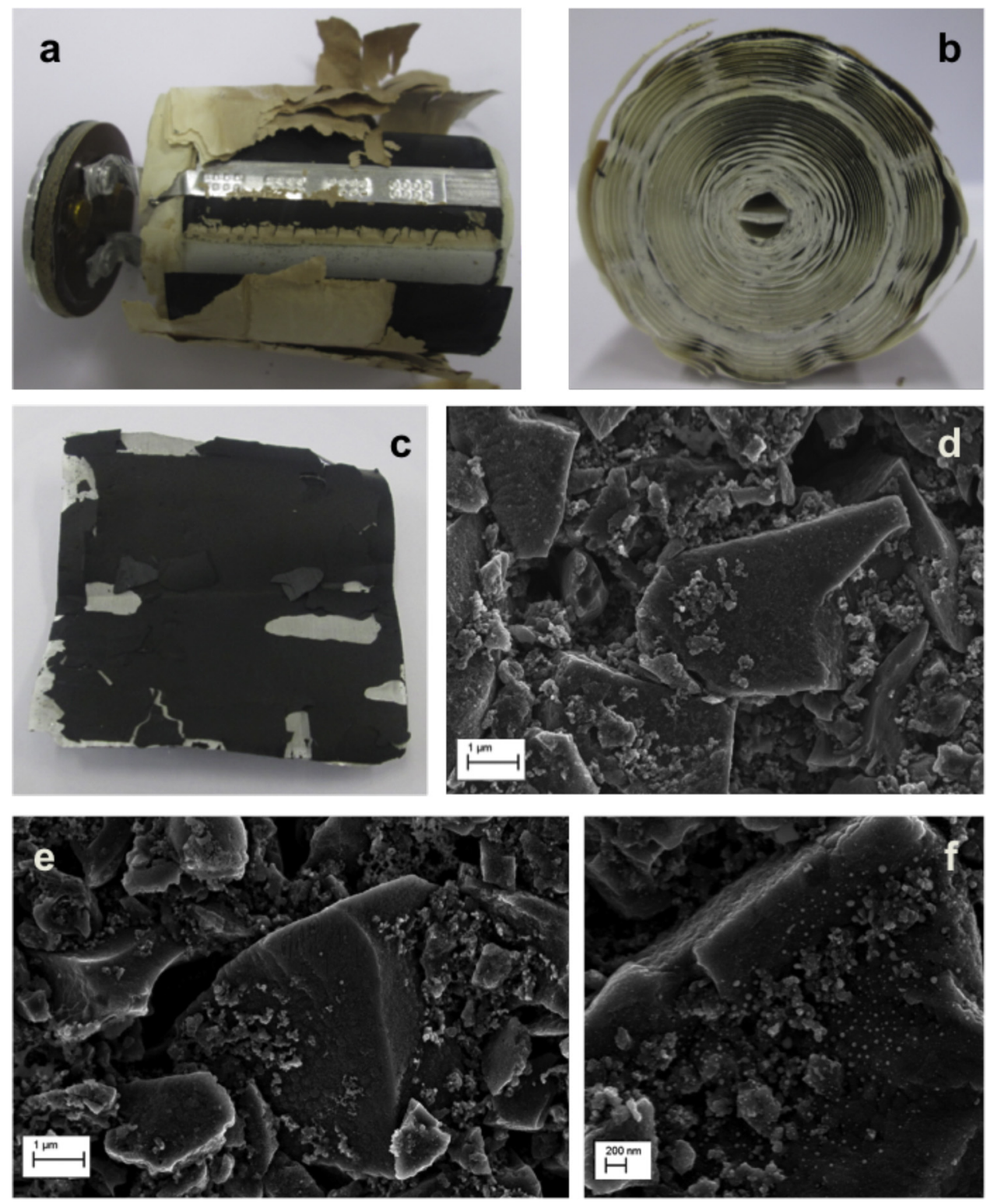

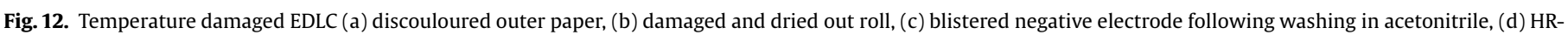
SEM of negative electrode, (e) HR-SEM of positive electrode, (f) nanoparticles evident on the surface of larger carbon particles of positive electrode. 
In order to physically explain the above experimental findings, it is important to underline that during a PC and LE ageing test there is a certain degree of solvent evaporation thus changing the composition of the electrolyte. Some of the evaporated solvent leaks from the SC which is not totally hermetic, and some of it remains inside the SC creating an overpressure. This overpressure modifies the contact between the electrode and electrolyte and leads to an additional $C_{\mathrm{SC}}$ decrease. This process depends strongly on the $T_{\mathrm{SC}}$ value and on the duration of the test. It is reasonable to assume that at the beginning of the whole ageing test and for short durations of LE phases (in our case shorter than $15 \mathrm{~h}$ ) the degradation of the electrolyte associated with the LE test is not so important and any benefit related to the extraction of current (during the PC test) is not producing any observable phenomena. After a certain threshold (in our case $15 \mathrm{~h}$ ) degradation of the electrolyte takes place and the increase of the temperature and removal of impurities associated with current extraction are temporarily compensating for the whole ageing process. For longer duration LE tests $(27 \mathrm{~h})$ the degradation of the electrolyte along with the number of micropores filled with impurities becomes important, consequently the extraction of current involves an important $C_{\mathrm{SC}}$ increase.

iii) If the $T_{\mathrm{SC}}$ is equal to $45^{\circ} \mathrm{C}$, once the $\mathrm{PC}$ is restarted, the first sub-phase associated to the recovering phenomena always takes place: the $C_{\mathrm{SC}}$ starts to increase until it reaches a
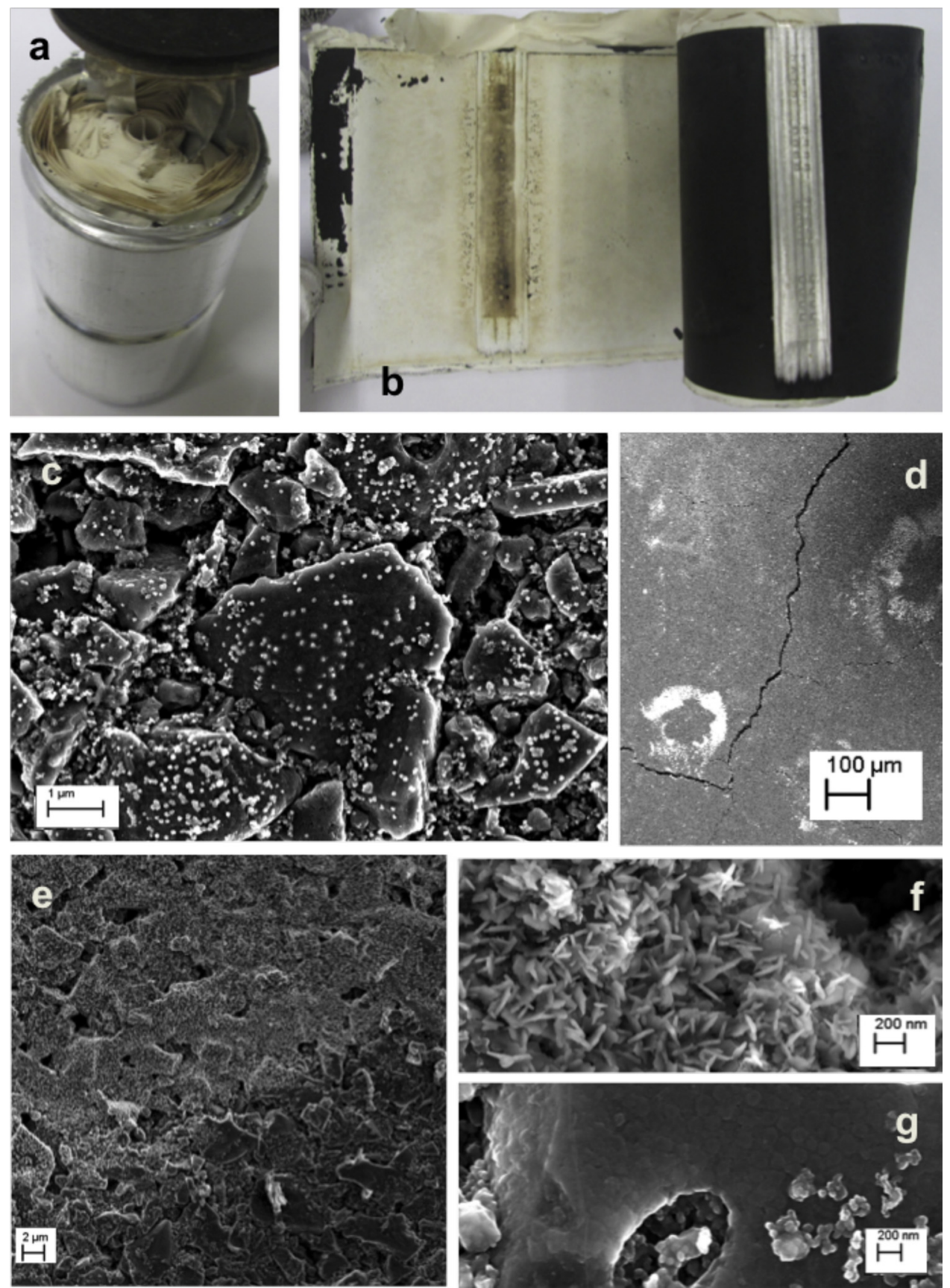

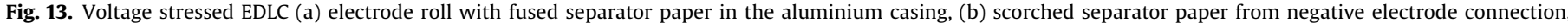

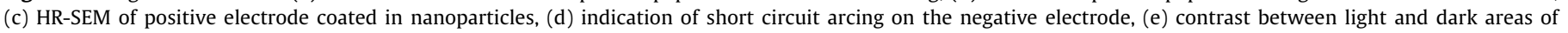
negative electrode, (f) high magnification of light area of the negative electrode, (g) film-like deposit on the negative electrode surface. 
maximum value. At the beginning of the test, during the first transition this phase holds for just $3 \mathrm{~h}$. For the other transition, since the degradation of the electrolyte is accelerated (the $T_{\mathrm{SC}}$ is higher) the current extraction temporarily benefits the SC. The $C_{\mathrm{SC}}$ increase is relatively higher than that evaluated at the same transition at $25^{\circ} \mathrm{C}$. This can be explained with a lower SC SoH involving a higher regeneration with current extraction.

It is worth nothing that the available literature (in particular, [6]) has been detected already a recovering phase during the transition PC to LE when the SC is cycled with its nominal current value and with current profiles representative of steady-state operations. On the contrary, in our study we have defined PC stresses with current profiles reflecting typical operating conditions of these devices (i.e. high dynamic pulsed currents).

\section{Post-mortem examination of the SC after overtemperature, overvoltage stress tests}

\subsection{Analysis of undamaged SC}

Following the PC and LE analysis of the SCs a post-mortem study of the stressed samples was undertaken. For the undamaged SCs the electrodes were tightly bound in a roll, and the outer paper was off white (Fig. 11a-c). The roll was saturated with the electrolyte to the centre and there was no free liquid. A section of each electrode was cut and washed 6 times in pure acetonitrile to remove the salt. The samples were then examined using high-resolution scanning electron microscopy (HR-SEM). As shown in Fig. 11d and e the electrode surface is highly porous and diverse. There is a mixture of large particles of activated carbon a few micrometers in diameter and smaller particles below $100 \mathrm{~nm}$ diameter that are the conductivity modifier, carbon black. The larger activated carbon particles also contain a nanoporous structure. In some areas a film or thick layer is also evident (Fig. 11f), which may be attributed to the polymer binding the carbon materials. There were no discernable differences between the positive and negative electrodes.

\subsection{Overtemperature stresses}

Acetonitrile has a boiling point of $82^{\circ} \mathrm{C}$. Consequently the high temperature stress of the SC would have boiled and evaporated the solvent within the cell, potentially increasing the concentration of the electrolyte until the point of salting out due to the loss of solvent. The ionic mobility of the electrolyte salts would also be improved due to a decrease in viscosity thus increasing the capacitance of the device.

Following over-temperature stress the vent cap of the SC was bulging but not perforated. This indicated that an overpressure had built-up in the capacitor during the stress procedure, corroborating the assumptions made in Section 2.1 to justify the $C_{\mathrm{SC}}$ fading. The outer paper of the central roll was severely discoloured to a yellow-brown, and was very dry and brittle (see Fig. 12). The discolouration became less pronounced towards the centre until the normal off-white separator paper was found, which was saturated with electrolyte.

Samples of the positive and negative electrodes were washed in acetonitrile in preparation for SEM analysis. During this procedure a number of observations were made (i) the negative electrode was found to blister and crack, (ii) the carbon detached from the aluminium backing, (iii) the backing electrode had severely oxidised and (iv) the positive electrode appeared undamaged by the washing procedure. In this respect, we can assume that the carbon detaching from the aluminium backing and the oxidisation of the current collector considerably raises the ESR, as observed in Section 2. The adhesion of the current collector to the electrode materials (i.e. aluminium to carbon) is crucial to the performance of a capacitor, determining a large aspect of the overall ESR. Furthermore, aluminium oxide is a poorly conducting material, and has a high electrical resistance. Kurzweil and Chwistek [17] noted the destruction at elevated temperatures of the typical aluminium material used as the current collector in commercial SCs. The aggressive fluoride anion in the electrolyte was found to react with the aluminium to form fluoroxy aluminate complexes.

SEM observed no major difference between these and the undamaged electrodes however, with no notable change in the porosity of the carbon surface (Fig. $12 \mathrm{~d}$ and e). In some areas of the positive electrode there was a covering of small nano droplets or particles. These melted rapidly underneath the electron beam of the SEM, and energy dispersive X-ray spectroscopy (EDX) analysis identified them as carbon based. It is possible these were electrolyte impurities or related to the polymer binding of the carbon electrode.

\subsection{Overvoltage stresses}

The overvoltage stressed SC was severely damaged following cycling. The main macroscopic observations were (i) the unit rattled when shaken, (ii) no electrical connection could be made, (iii) the pressure vent had expanded but had not perforated; (iv) the over-voltage stress greatly expanded the volume of the roll (Fig. 13a).

On extracting the roll, the outer paper was yellowed, very brittle and dry. On unravelling the electrodes scorch marks were evident on the separator paper where the negative aluminium ribbon had been in contact (Fig. 13b). The capacitor held very little electrolyte all the way to the centre of the roll. The positive electrode exhibited a patchy, exfoliated surface, and had become stuck to the separator paper in places. The paper was very dry and had fused to other sheets at the ends of the roll. On washing a sample of each electrode in acetonitrile the negative electrode became scaled and flaky, again indicating a loss of adhesion, but not to the same extent as for the over-temperature stressed electrode. Furthermore, a yellow tinge became evident in the acetonitrile (see Fig. 14). The unknown compound was analysed using $1 \mathrm{H}-\mathrm{NMR}$ and no other compounds were observed when compared to a solution of just TEA $\mathrm{BF}_{4}$ in $\mathrm{CD}_{3} \mathrm{CN}$, strongly suggesting the yellow colour was caused by triethylamine and likely other $N$-alkyl amines (Fig. 14). Triethylamine develops a yellow tinge due to impurities on long standing in air. The formation of triethylamine indicates a break down of the electrolyte, which would gradually decrease the conductivity of the electrolyte and also introduce parasitic faradaic processes that result in capacitance fade. This would be observed as a gradual decrease in the $C_{\mathrm{SC}}[7]$.

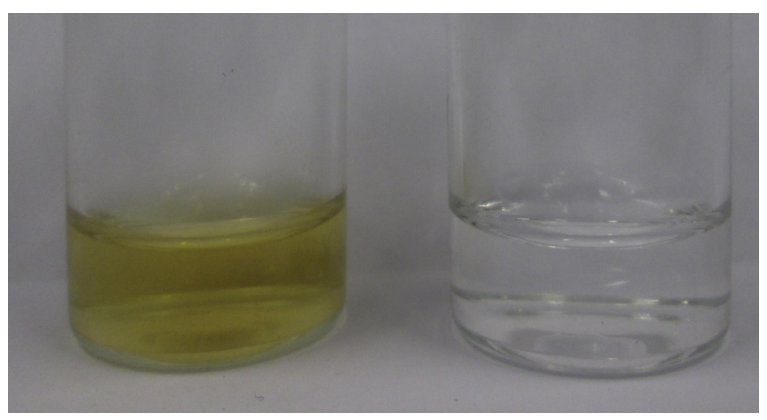

Fig. 14. Right: acetonitrile with $1 \mathrm{M} \mathrm{TEA} \mathrm{BF}$, left: acetonitrile from washing the negative electrode of the voltage damaged EDLC. Indicative of the presence of impure triethylamine as well as the TEA $\mathrm{BF}_{4}$. 
SEM analysis of the voltage stressed electrodes revealed a variety of morphological and surface changes. On one side of both the positive and negative electrodes a uniform deposition of round nanoparticles was observed (Fig 13c). EDX analysis revealed that the particles were carbon based, suggesting they were formed of the organic material in the capacitor such as a component of the polymer binder. One possibility is butadiene-styrene, a commonly used rubber polymer used in the binder with a regular particle size of approximately $130 \mathrm{~nm}$. As these particles have been seen to different extents on all damaged and undamaged electrodes, it strongly suggests they are a feature of the binder material. Note the exact composition of the binder is unknown due to IP restrictions, however certain patents reveal likely components of the material [18].

On the other side of the positive electrode sample the surface was not covered in particles, but was found to be considerably cracked on the nanoscale, with sharp, exfoliated graphite particles distributed across the surface (Fig. 16). These cracks are due to the evolution of gases underneath the surface layers of the activated carbon electrode, specifically the evolution of $\mathrm{O}_{2}$ from residual water oxidation, and the decomposition of the electrolyte and solvent at high voltages [19].

Further morphological differences were observed on the negative electrode of the voltage stressed SC. Fig. 13d is a low magnification image of the electrode surface, and shows large cracks that are typical across the whole sample. The SEM also revealed distinct areas on the electrode surface area indicative of localised damage rather than whole electrode modification. The areas were also observed to a lesser extent on the positive electrode surface. Fig. 13e straddles the boundary of the dark and white patch with Fig. 8f showing the dense crystalline coating across the "white" area. Unlike the nanoparticles shown in Fig. 8c, these crystal structures did not melt or evaporate under the electron beam. EDX analysis found them to be composed of aluminium and oxygen, typical of $\mathrm{Al}_{2} \mathrm{O}_{3}$ (Fig. 15). The formation of such an oxide, and the distinct shape of the patches suggest that electrical arcing occurred between the positive and negative electrode. Electric arcing occurs in the gas-filled space between two conductive electrodes and it results in a very high temperature, capable of melting or vaporising most materials. It is worth noting that within the confines of the capacitor with a high over pressure of acetonitrile vapor the probability of an arc forming between the overly charged electrodes is high. The formation of large areas of $\mathrm{Al}_{2} \mathrm{O}_{3}$, in addition to the cracking of the surface and loss of current collector-electrode contact would decrease the electroactive area of the negative electrode, thus greatly increasing the ESR of the capacitor. Furthermore, the ribbons of the current collector were clearly damaged by the voltage stress therefore raising the resistivity of the aluminum. These observations corroborate the findings in Section 2 in which surges in temperature were periodically observed, and the ESR rapidly increased.

In other areas of the negative electrode the formation of a film was evident across the activated carbon (Fig. 8g) Film formation at high voltages was previously noted by Ishimoto et al. [19], and attributed to the decomposition of the electrolyte and polymerisation on the modified surface. Zhu et al. [21] also concluded extensive polymerisation on the carbon surface during high voltage ageing. EDX analysis of the film coated surface found a considerable increase in the fluorine and oxygen levels compared to the electrodes of an undamaged electrode. Based on the observations of reference [21] it may be postulated that the polymerisation is due to decomposition of either the $\mathrm{BF}_{4}^{-}$anion of the electrolyte, or indeed the binder, which in many SCs is a fluorinated polymer. Such a film would undoubtedly affect the penetration of electrolyte into the pores of the carbon surface and greatly diminish the capacitance of the material.

\subsection{Voltammetric study of electrode performance}

In order to determine the behaviour of the activated carbon electrodes in controlled conditions and at specific potentials, samples of the electrodes were cut to a specific size and studied in conventional two and three electrode set-ups. The electrolyte was $1 \mathrm{M}(25 \%) \mathrm{TEABF}_{4}$ in acetonitrile $(\mathrm{AcN})$. The viscosity of the electrolyte was found to vary considerably with respect to the concentration of electrolyte and temperature. At $23^{\circ} \mathrm{C}$ the viscosity of $25 \% \mathrm{TEABF}_{4}$ was determined to be $0.66 \mathrm{mPas}$ which decreased to $0.4 \mathrm{mPas}$ at a temperature of $50^{\circ} \mathrm{C}$. At room temperature $10 \%$ $\mathrm{TEABF}_{4}$ had a viscosity of $0.51 \mathrm{mPas}$ whereas pure acetonitrile has a viscosity of $0.35 \mathrm{mPas}$ at $25^{\circ} \mathrm{C}$.
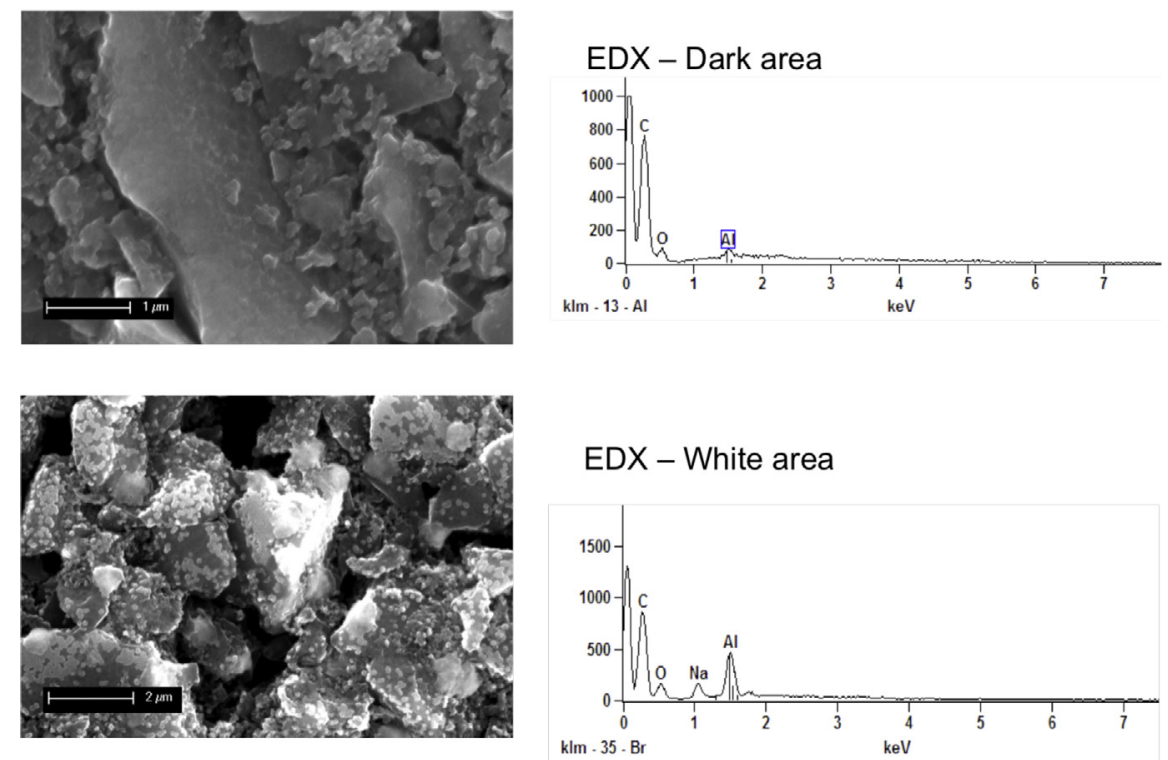

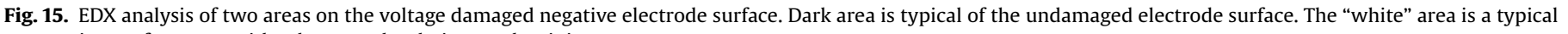
contrasting surface area with a large peak relating to aluminium. 

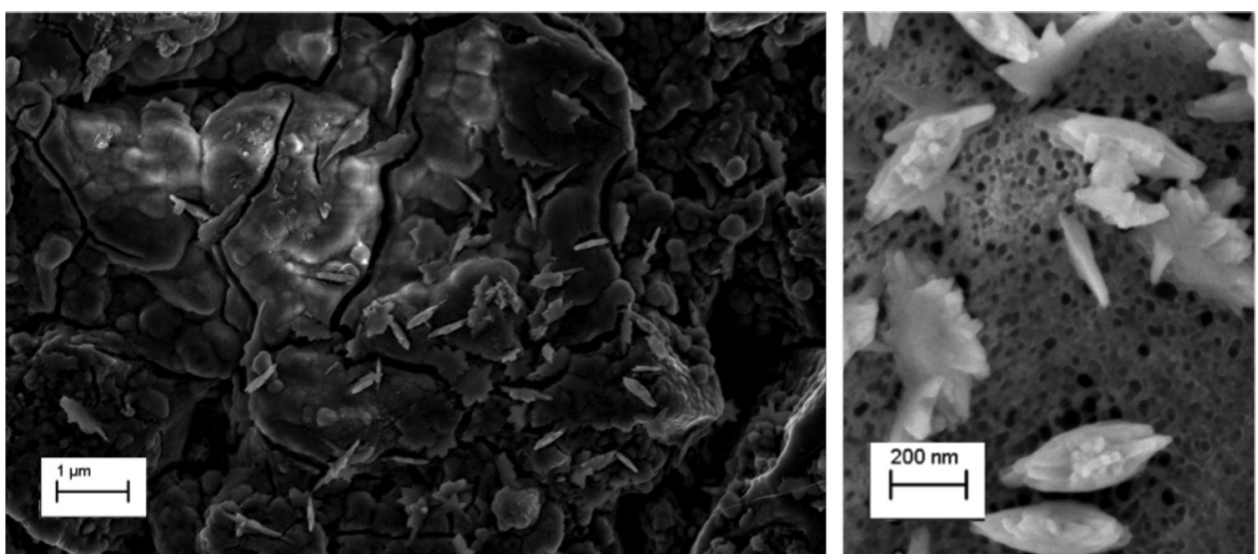

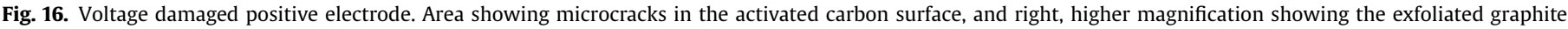
particles. (For interpretation of the references to colour in this figure legend, the reader is referred to the web version of this article.)

Fig. 16a and b shows the voltammetric response of the carbon electrode when the working potential was gradually decreased to $-3 \mathrm{~V}$ or increased to $4 \mathrm{~V}$ vs. $\mathrm{Ag} / \mathrm{AgBF}_{4}$ electrode. A detrimental effect on the capacitance is evident from $-1.6 \mathrm{~V}$, with a potential limit observed at $-2.8 \mathrm{~V}$. For the positive potential sweeps, up to $2.4 \mathrm{~V}$ the electrode performs well, but a rapid decline in capacitance occurs at higher potentials. These are typical potential windows for carbon in wet acetonitrile.

Finally a 2-electrode arrangement was studied, imitating the SC cell, with two activated carbon electrodes of equal geometric area. In Fig. 16c a decline in capacitance is observed from a cell potential of $3.4 \mathrm{~V}$ (in red). (Note that the potentials here are for the whole cell, whereas the voltages in a 3-electrode system are the working electrode potentials only). This corroborates the performance observed in the power cycling tests at overvoltage, in which rapid $C_{\mathrm{SC}}$ fading along with a rapid increase of ESR have been observed from 3.5 $\mathrm{V}$ and above (Table 3 and Fig. 6). It suggests that from cell voltages of $3.4 \mathrm{~V}$ the electrodes are changing detrimentally, either oxidising and being damaged, losing their porosity and therefore capacitive volume, or a chemical reaction is polymerising the electrolyte and/or binder to block the surface of the electrodes.

Interestingly, the positive electrode greatly expanded during the cycling experiments, as shown in Fig. 13. This clearly indicates that the positive electrode undergoes extensive physical changes during potential cycling to high voltages that would greatly influence the capacitance of the material. This behaviour was noted in a similar experiment by Ishimoto et al. [19] in which the activated carbon particles of the anode were shown to swell and exfoliate from moderately high voltages. Although the positive electrode of the SC was not found to have expanded thin layers of exfoliated graphite were observed on the separator paper. The assembly of the compressed roll may have simply prevented the electrode expanding in the manner shown in Fig. 9d.

In this study the examined SCs were exposed to every temperature or voltage stress. Consequently the experimental macro and micro observations cannot be attributed to a specific condition (i.e. specific voltage or temperature). Further studies to identifying at what point physical modification of the electrodes and damage to the components occurs would also be interesting. However, it is possible to distinguish between faults associated to over-temperature vs. over-voltage stresses. In the following two sections, the correlation between macroscopic and microscopic observations is discussed and a specific fault diagnosis algorithm is proposed.

\section{Correlations between macroscopic and microscopic experimental observations}

This section aims to summarise the links between macroscopic and microscopic observations discussed in the previous sections (Table 6). Table 4 lists the different observations made regarding the ESR, $C_{\mathrm{SC}}$ and $V_{20}$ trends for the over-temperature and overvoltage stress tests. For each claim, the associated microscopic observations are reported.

Based on the post-mortem analysis and Figs. 11-17, it is reasonable to assume that the SCs exhibit a robust behaviour after over-temperature stress tests, conversely, they appear quite sensitive to over-voltage exposure. Fig. 18 shows a comparison

Table 6

Links between macroscopic and microscopic fault diagnosis observations.

\begin{tabular}{|c|c|c|}
\hline Type of fault & Macroscopic observations & Microscopic observations \\
\hline Overtemperature & $\begin{array}{l}\text { ESR increase: } \\
\text { possible changes in electrolyte properties, impurities } \\
\text { production, internal overpressure } \\
C_{\mathrm{SC}} \text { increase } \\
V_{20}\end{array}$ & $\begin{array}{l}\text { Carbon detaching from the aluminum backing, severe oxidation of the aluminium } \\
\text { backing; impurities production } \\
\text { Not available } \\
\text { Related to the previous findings }\end{array}$ \\
\hline Overvoltage & $\begin{array}{l}\text { Large increase in ESR: } \\
\text { Viscosity electrolyte changes, over temperatures, possible } \\
\text { micro short-circuits, possible electrolyte decomposition } \\
C_{S C} \text { important decrease: } \\
\mathrm{S}_{\mathrm{SC}} \text { modifications, changes in electrolyte properties } \\
V_{20} \text { important decrease: } \\
\text { Over temperature modifying the charge redistribution, } \\
\text { viscosity changes }\end{array}$ & $\begin{array}{l}\text { Porosity changes due to electrode oxidation, short-circuit arcing on the negative } \\
\text { electrode, film formation on the negative electrode surface } \\
\text { Negative effect on the capacitance from a cell potential of } 3.4 \mathrm{~V} \text { (voltammograms) } \\
\text { arcing cause overpressure, viscosity changes and parasitic faradaic currents due to } \\
\text { impurities production } \\
\text { Arcing causes over temperature, electrolyte decomposition modifying its viscosity, } \\
\text { surface modification coverage of } \mathrm{Al}_{2} \mathrm{O}_{3}\end{array}$ \\
\hline
\end{tabular}


a

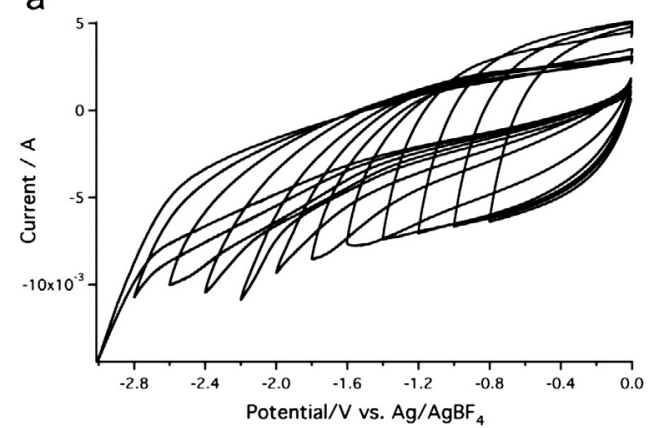

C

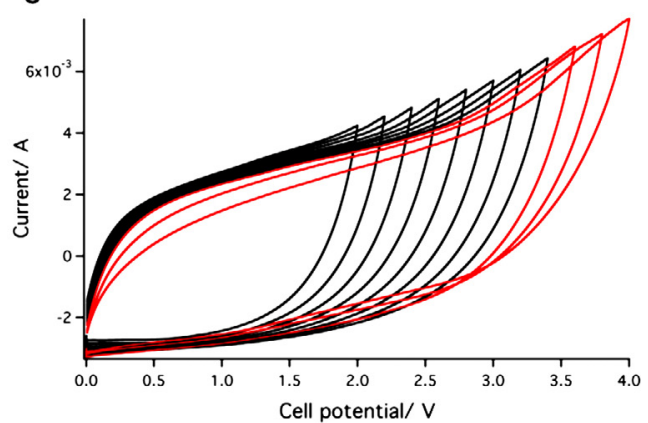

b

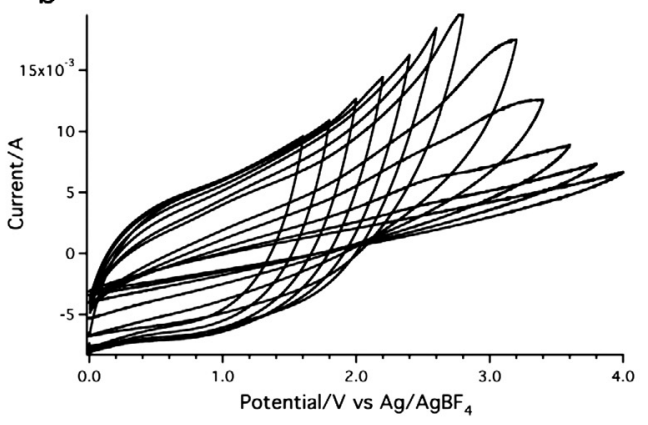

d

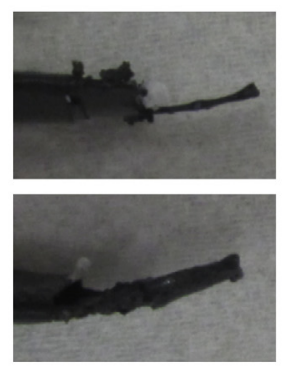

\section{Positive}

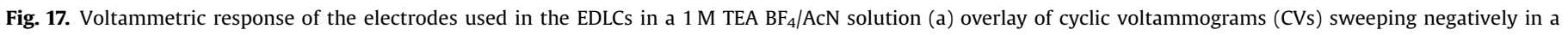

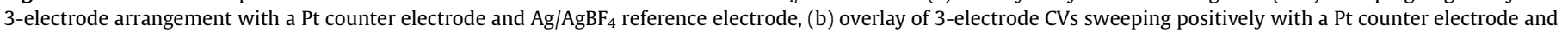

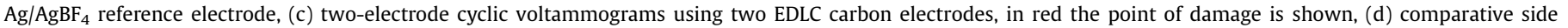
view photographs of the positive and negative electrodes following 2-electrode cycling.

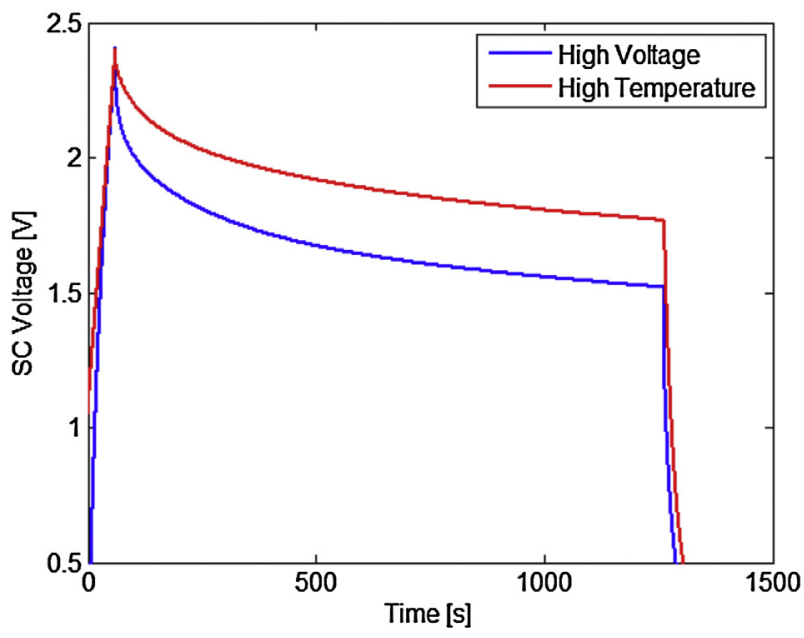

Fig. 18. Comparison between the worst overtemperature and overvoltage test

between SC decay voltages related to the extreme over-temperature and over-voltage tests, namely $130^{\circ} \mathrm{C}$ after $72 \mathrm{~h}$ and $4 \mathrm{~V}$ during cycling. As it can be observed, the over-voltage effect is more important than the over-temperature one. ${ }^{3}$

\section{Proposed fault indicators}

The experimental findings illustrated before have suggested specific fault indicators that can be structured to define an algorithm capable of detecting consecutive over-temperatures or over-voltages faults in SCs. Indeed, it is possible to detect specific trends of the four parameters: ESR, $C_{\mathrm{SC}}, V_{20}$ and high pulsed power capability (HPC, defined below in Eq. (2)) having distinct behaviours after over-temperature, over-voltage and nominal use (Fig. 19).

The proposed algorithm is composed of fours different steps where comparisons between the values of the above parameters with threshold values, which correspond to a potential fault diagnosis, are made. In this respect, we henceforth define as $E_{\mathrm{SR}, 0}$, $C_{\mathrm{SC}, 0}$ and $V_{20,0}$ as the values of ESR, $C_{\mathrm{SC}}, V_{20}$ associated to the undamaged targeted SC (for instance, the starting values assumed for an un-cycled SC). The proposed algorithm uses as first entry the ESR value in view of its easy experimental assessment. In what follows the logic of the proposed algorithm is illustrated.

1) The initial step requires evaluating the ESR of the targeted SC. Once this measurement is performed its value has to be compared with ESR,o. The following cases are possible:

a) ESR increase is in the range of $90-1100 \% \mathrm{ESR}, 0$;

b) ESR increase is in the range of $1.1-1.9 \%$ of ESR, ${ }_{0}^{4}$;

If condition 1-a is satisfied one may assume that a consecutive overvoltage fault occurred in the targeted SC.

If condition 1-b is satisfied it is not possible to distinguish between the two fault types and further investigations are needed (step 2).

2) The second step evaluates the $C_{\mathrm{SC}}$ (this measurement does not require a complex procedure). Once this measurement is performed it is compared with $C_{\mathrm{SC}, 0}$. The following cases are possible:

\footnotetext{
${ }^{3}$ The aim of the comparison shown in Fig. 18 is not to detect a correlation between temperature stresses and voltage ones but to assess and compare the SC behaviours with respect to the worst overtemperature and overvoltage stressing
} conditions.

\footnotetext{
${ }^{4}$ It is worth nothing that the SCs performances are also normally fading due to the nominal usage of the device. Therefore, in order to misestimate the presence of a fault, the values of ESR, 0 and $C_{\mathrm{SC}, 0}$ have to be updated along the usage of the device.
} 


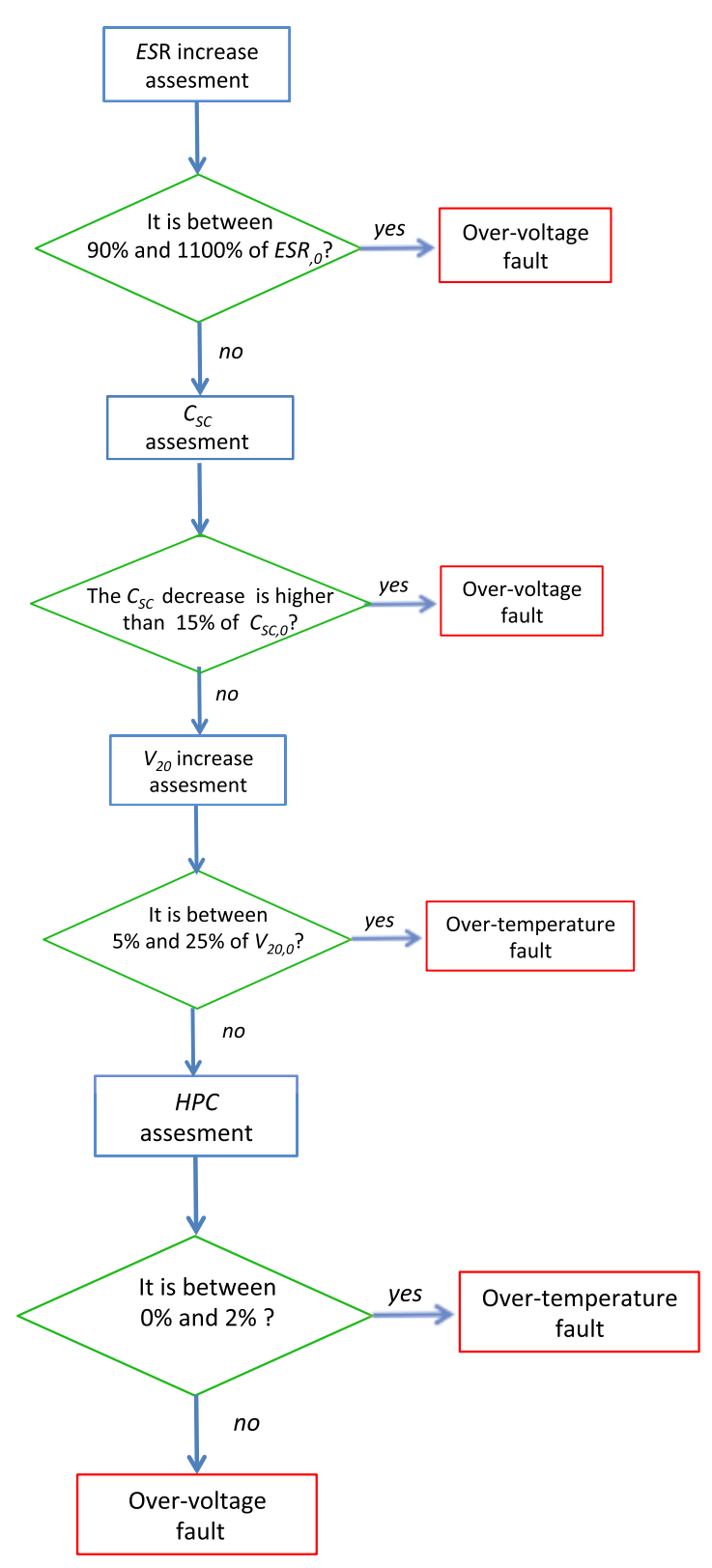

Fig. 19. Proposed fault-diagnosis algorithm for SCs.

a) $C_{\mathrm{SC}}$ decrease is greater than $15 \%$ times $C_{\mathrm{SC}, 0}$;

b) $C_{\mathrm{SC}}$ decrease is between $2 \%$ and $10 \%$ times $C_{\mathrm{SC}, 0}$;

If condition $2-\mathrm{a}$ is satisfied, one can assume that a consecutive overvoltage fault occurred to the targeted SC.

If condition 2-b is satisfied it is not possible to distinguish between the two fault types and further investigations are needed (step 3 ).

3) The third step evaluates $V_{20}$. Once this measurement is performed, its value is compared to $V_{20,0}$. The following cases are possible

a) $V_{20}$ decrease is between $5 \%$ and $25 \%$ of $V_{20,0}$;

b) $V_{20}$ decrease is between $1 \%$ and $4 \% V_{20,0}$;

If condition 3-a is satisfied, it is possible to assume that a consecutive over-temperature fault occurred in the targeted SC. If condition 3-b is satisfied, it is not possible to distinguish between the two fault types and further investigations are needed (step 4).

4) The last step evaluates the HPC. This measurement requires just a few seconds but also requires an electronic load and a current sensor of bandwidth equal to $100 \mathrm{kHz}$. Once the current delivered by the SC has been recorded it has to be compared with the current profile delivered by the undamaged SC. This comparison is based on the following equation:

$\mathrm{HPC}=\max \left[\left|\frac{i_{\mathrm{ref}}(t)-i_{\mathrm{SC}}(t)}{i_{\mathrm{REF}}(t)}\right|\right] 100$

In the previous definition of HPC, $i_{\text {ref }}(t)$ represents the high-pulsed current delivered by the un-cycled SC; $i_{\mathrm{SC}}(t)$ represents the current delivered by the damaged SC.

The following cases are possible:

a) HPC is between 0 and $2 \%$;

b) HPC is above $5 \%$;

If condition 4-a is satisfied, it is possible to assume that a consecutive over-temperature fault occurred to the targeted SC. If condition 4-b is satisfied, it is possible to assume that a consecutive over-voltage fault occurred to the targeted SC. The fault diagnosis process is completed.

Fig. 11 illustrates schematically the proposed algorithm. The blue boxes show the required experimental procedure to perform fault diagnosis. The green boxes show the ranges of each fault indicator, for which it is possible to perform the fault diagnosis. The red boxes indicate the fault diagnosis conclusions. Note that, the proposed algorithm could start with any of the fault indicators among the four we analysed.

If none of the different conditions is fulfilled, based on the proposed experimental analysis, it means that the targeted SC has not been stressed neither with overvoltage and neither with consecutive overtemperature stresses.

\section{Conclusions}

This work has described an experimental study aimed at:

i) analysing the fault diagnosis of SCs during high temperature and high voltage stress;

ii) discussing the SC state of health and $C_{S C}$ evolution during combined PC and LE tests at different temperatures.

The evolution of the SC characteristic parameters (i.e. ESR and $C_{\mathrm{SC}}, V_{20}$ ) under extreme conditions of high temperatures (up to $130^{\circ} \mathrm{C}$ ) and voltages (up to $4 \mathrm{~V}$ ) have been analysed in order to infer characteristic behaviours. The performances evaluation has been computed in term of increase of ESR, decrease of $C_{\mathrm{SC}}$, increase of the decay voltage and capability to deliver high pulsed current. Their performances fading have been investigated in two specific cases: during subsequent and single stresses.

For subsequent overtemperature stresses, the obtained results show that SCs exhibit a robust behaviour. Namely, the ESR of the targeted SC does not increase considerably despite the extreme high value of the temperature, the $C_{\mathrm{SC}}$ increase its value and the SC is always able to deliver high pulsed currents (up to 5 times the nominal value). Conversely, these devices appear quite fragile against subsequent overvoltage exposures. Indeed, the ESR of the targeted SC can even be 11 times higher the initial value, the decrease of the $C_{\mathrm{SC}}$ reaches the $20 \%$ of the initial value and the SC is no more able to deliver high-pulsed currents (it can still deliver the nominal one).

We have investigated also the effects single overtemperature and overvoltage stresses characterised by the same magnitudes as in the consecutive ones. The results are:

i) For single overtemperature stresses the changes in $C_{\mathrm{SC}}$, ESR and $V_{20}$ are quite mitigated compared to those observed for 
consecutive overtemperature stresses. The increase of ESR is mitigated of $50 \%$ and the decrease of $V_{20}$ is mitigated of $20 \%$.

ii) For single overvoltage stresses the changes in $C_{\mathrm{SC}}$, ESR and $V_{20}$ are also quite mitigated compared to those observed for consecutive overvoltage stresses. The increase of ESR is mitigated from $30 \%$ up to $900 \%$ and the decrease of $V_{20}$ is mitigated of $15 \%$. The main difference is associated to the capability of the tartegeted SC to still deliver high pulsed current event after the application of a $4 \mathrm{~V}$ voltage.

Specific physical hypothesis have been proposed to justify the trend of the three SC characteristic parameters (i.e. ESR and $C_{\mathrm{SC}}$, $V_{20}$ ). These were supported by the post-mortem analysis performed on the damaged SCs. In particular, post-mortem examination of the stressed capacitors found considerable variation in the electrode surface morphology relative to the undamaged electrodes. The most pronounced damage was observed in the voltage stressed cell, in which nanoparticle deposition, suspected electrical arcing, and polymer film formation were observed. Temperature damage was less extensive, but severely oxidised and damaged the negative electrode in particular.

Based on the above results, a method was proposed to detect the type of faults based on macroscopic fault indicators (ESR and $C_{\mathrm{SC}}, V_{20}$ and $\mathrm{HPC}$ ), providing an algorithm that represents a method to perform online and real-time diagnosis. These results and the associated algorithm may be used to improve existing analytical SC ageing models to enable online and real-time state of health estimations.

Concerning the second main outcome of the paper, namely the SOH estimation during combined PC and LE stress, a new recovering phase along with an accelerated ageing phase have been identified. In summary, the recovering phase, characterised by a temporary increase of the $C_{\mathrm{SC}}$, appears at each transition from LE to PC. The duration and magnitude of the $C_{\mathrm{SC}}$ increase depends on $T_{\mathrm{SC}}$ and on the duration of the previous stress. The accelerated ageing phase, characterised by a temporary decrease of the $C_{\mathrm{SC}}$, appears at each transition from PC to LE. The duration and magnitude of the CSC decrease depends strongly on $T_{\mathrm{SC}}$ and on the duration of the previous stress. This specific phase, along with the recovering one, have been investigated for three different temperatures, the nominal $T_{\mathrm{SC}}\left(25^{\circ} \mathrm{C}\right)$, a slightly higher $T_{\mathrm{SC}}\left(35^{\circ} \mathrm{C}\right)$ and a moderately high $T_{\mathrm{SC}}\left(45^{\circ} \mathrm{C}\right)$. The main findings are (i) a high-pulsed power current extraction can be used for temporary increases of $C_{\mathrm{SC}}$ and this increase is more important at lower temperatures (i.e. $25^{\circ} \mathrm{C}$ ); (ii) during the transition $\mathrm{PC} \rightarrow \mathrm{LE}$ stress there in a decrease of $C_{\mathrm{SC}}$ more significant at higher temperatures (i.e. $45^{\circ} \mathrm{C}$ ).

\section{Acknowledgments}

The authors thankfully acknowledge the Swiss Innovation and Technology Committee (CTI) since the scientific activity described in this paper is framed within the context of the researches of the Swiss Competence Center for Energy Research "FURIES" (Future Swiss Electrical Infrastructure). The authors thankfully acknowledge M. Robert and M. Burri for their help in carrying out the experimental investigations. Also, the authors would like to thank E. Smirnov for additional SEM imaging and useful discussions.

\section{References}

[1] A. Oukaoura, B. Tala-Ighila, M. AlSakkab, H. Gualousa, R. Gallayc, B. Boudarta, Calendar ageing and health diagnosis of supercapacitor, Electr. Power Syst. Res. 95 (2013) 330-338.

[2] E. Brian Conway, W.G. Pell, T.-C. Liu, Diagnostic analyses for mechanisms of self-discharge of electrochemical capacitors and batteries, J. Power Sources 65 (1997) 53-59.

[3] A.G. Pandolfo, A.F. Hollenkamp, Carbon properties and their role in supercapacitors, J. Power Sources 157 (2006) 11-27.

[4] T. Nishida, Y. Tashiro, M. Yamamoto, Phsycal and electrochemical properties of 1-alkyl-3-methylimidazolium tetrafluoroborate for electrolyte, J. Fluorine Chem. 120 (2003) 135-141.

[5] M. Marracci, B. Tellini, M. Catelani, L. Ciani, Ultracapacitor degradation state diagnosis via electrochemical impedance spectroscopy, instrumentation and measurement, IEEE Trans. PP (99) (2015) 1.

[6] R. Chaari, O. Briat, J.Y. Deletage, J. Vinassa, Performances regeneration of supercapacitors during accelerated ageing tests in power cycling, Power Electron. Appl. (EPE 2011) (2011) 1-7 (Aug. 30 2011-Sept. 1 2011).

[7] D. Torregrossa, M. Paolone, Novel Experimental Investigation of Supercapacitor Ageing during Combined Life-Endurance and Power-Cycling Tests, IECON, Vienna, 2013 (November).

[8] O. Bohlen, J. Kowal, D.U. Sauer, Ageing behaviour of electrochemical double layer capacitors Part II. Lifetime simulation model for dynamic applications, J. Power Sources 173 (2007) 626-632.

[9] O. Briat, J.-M. Vinassa, N. Bertrand, H. El Brouji, J.-Y. Dele’tage, E. Woirgard, Contribution of calendar ageing modes in the performances degradation of supercapacitors during power cycling, Microelectron. Reliab. 50 (2010) 1796-1803.

[10] R. Chaari, O. Briat, J.Y. Deletage, J. Vinassa, Performances regeneration of supercapacitors during accelerated ageing tests in power cycling, Power Electron. Appl. (EPE 2011) (2011) 1-7 (Aug. 30 2011-Sept. 1 2011).

[11] N. Omar, H. Gualous, J. Salminen, G. Molder, A. Samba, Y. Firouz, M. Monem, P. Van den Bossche, J. Van Mierlo, Electrical double layer capacitors: evaluation of ageing phenomena during cycle life testing, J. Appl. Electrochem. 44 (2014) 509-522.

[12] A. Yu, V. Chabot, J. Zhang, Electrochemical Supercapacitors for Energy Storage and Delivery: Fundamentals and Applications, CRC Press, 2013 (April 9).

[13] D. Torregrossa, M. Bahramipanah, R. Cherkaoui, M. Paolone, Experimental evidences of redistribution phenomenom in supercapacitors and consequent dynamic modeling improvement, Power Tech Conference, 16-20 June, IEEE 2013.

[14] C. Lin, B. Popov, H.J. Ploehnz, Modeling the effects of electrode composition and pore structure on the performance of electrochemical capacitors, J. Electrochem. Soc. 149 (2) (2002) 167-175

[15] J. Vetter, P. Novak, M. Wagner, C. Veit, K. Moller, J. Besenhard, M. Winter, M. Wohlfahrt-Mehrens, C. Bogler, A. Hamouche, Ageing mechanism in lithium-ion batteries, J. Power Sources 147 (2005) 269-281.

[17] P. Kurzweil, M. Chwistek, Electrochemical stability of organic electrolytes in supercapacitors: spectroscopy and gas analysis of decomposition products, J. Power Sources 176 (2) (2008) 555-567 (1 February).

[18] Y.J. Jung, K.S. Yoo, J.H. Choi, K.H. Kwon, Y.H. Kim, Electrode pour condensateur électrochimique et son procédé de préparation, WO 2009091102A1.

[19] S. Ishimoto, Y. Asakawa, M. Shinya, K. Naoi, Degradation responses of activated-carbon-based EDLCs for higher voltage operation and their factors batteries and energy storage, J. Electrochem. Soc. 156 (7) (2009) A563-A571.

[21] M. Zhu, C.J. Weber, Y. Yang, M. Konuma, U. Starke, K. Kern, A.M. Bittner Chemical and electrochemical ageing of carbon materials used in supercapacitor electrodes, Carbon 46 (2008) 1829-1840. 